\title{
in tinbergen
}

TI 2020-054/II

Tinbergen Institute Discussion Paper

\section{Compromising between the proportional and equal division values: axiomatization, consistency and implementation}

Zhengxing Zou ${ }^{1}$

Rene van den Brink ${ }^{2}$

Yukihiko Funaki3

\footnotetext{
${ }^{1}$ School of Economics and Management, Beijing Jiaotong University, Beijing, China, and Department of Economics, Vrije Universiteit Amsterdam, The Netherlands

2 Department of Economics, and Tinbergen Institute, Vrije Universiteit Amsterdam, The Netherlands

${ }^{3}$ School of Political Science and Economics, Waseda University, Tokyo, Japan
} 
Tinbergen Institute is the graduate school and research institute in economics of Erasmus University Rotterdam, the University of Amsterdam and Vrije Universiteit Amsterdam.

Contact: discussionpapers@tinbergen.nl

More TI discussion papers can be downloaded at https://www.tinbergen.nl

Tinbergen Institute has two locations:

Tinbergen Institute Amsterdam

Gustav Mahlerplein 117

1082 MS Amsterdam

The Netherlands

Tel.: +31(0)205984580

Tinbergen Institute Rotterdam

Burg. Oudlaan 50

3062 PA Rotterdam

The Netherlands

Tel.: +31(0)10408 8900 


\title{
Compromising between the proportional and equal division values: axiomatization, consistency and implementation
}

\author{
Zhengxing Zou ${ }^{* \dagger} \quad$ René van den Brink ${ }^{\ddagger} \quad$ Yukihiko Funaki ${ }^{\S}$
}

August 28, 2020

\begin{abstract}
We introduce a family of values for TU-games that offers a compromise between the proportional and equal division values. Each value, called an $\alpha$-mollified value, is obtained in two steps. First, a linear function with respect to the worths of all coalitions is defined which associates a real number to every TU-game. Second, the weight assigned by this function is used to weigh proportionality and equality principles in allocating the worth of the grand coalition. We provide an axiomatic characterization of this family, and show that this family contains the affine combinations of the equal division value and the equal surplus division value as the only linear values. Further, we identify the proportional division value and the affine combinations of the equal division value and the equal surplus division value as those members of this family, that satisfy projection consistency. Besides, we provide a procedural implementation of each single value in this family.
\end{abstract}

Keywords : Cooperative game; consistency; equal division value; proportional division value

${ }^{*}$ Department of Economics, Vrije Universiteit Amsterdam, De Boelelaan 1105, 1081 HV Amsterdam, The Netherlands. E-mail: zhengxingzou@126.com

${ }^{\dagger}$ School of Economics and Management, Beijing Jiaotong University, Beijing 100044, China

${ }^{\ddagger}$ Department of Economics, and Tinbergen Institute, Vrije Universiteit Amsterdam, De Boelelaan 1105, 1081 HV Amsterdam, The Netherlands. E-mail: j.r.vanden.brink@vu.nl

${ }^{\S}$ School of Political Science and Economics, Waseda University, 1-6-1 Nishi-Waseda, Shinjuku-Ku, Tokyo 169-8050, Japan. E-mail: funaki@waseda.jp 


\section{Introduction}

A situation in which players can generate certain worths from cooperation can be described by a cooperative game with transferable utility, or simply a TU-game or a game. It consists of a set of players and a characteristic function that specifies a worth for each coalition of players. The central question is how to allocate the worth of the grand coalition (consisting of all players) over the individual players. Undoubtedly, an extreme egalitarian value is the equal division value (characterized in van den Brink (2007)), which allocates the total worth equally among all players. As an alternative to this equality principle, the proportional division value (Zou et al., 2019) relies on the proportionality principle: it gives payoffs in proportion to players' stand-alone worth.

Proportionality and equality are fundamental principles in various allocation problems. Specifically, in bankruptcy problems where the available amount of a resource that is to be allocated is not enough to honor all players' claims on it, a pioneering work was done by O'Neill (1982) which shows that the proportional and equal rules are two prominent concepts both in practice and in the theory. Subsequently, some research has focused on comparing and characterizing different ways of compromising between the proportional and equal rules for bankruptcy problems and other related problems. The reader is referred to Moulin (1987); Giménez-Gómez and Peris (2014); Thomson (2015b), and to Thomson (2003, 2015a) for overviews of this literature. As shown in O'Neill (1982), a bankruptcy problem can be modeled as a TU-game. However, the sizable literature does not touch the issue of generalizing values that compromise between proportionality and equality principles for general TU-games.

In this paper, we introduce a family of values for TU-games that offers a simple yet flexible compromise between proportionality and equality principles. Our values, which we call $\alpha$-mollified values, contain convex combinations of the equal division value and the proportional division value ${ }^{1}$ introduced in Moulin (1987) for a special type of game. Specifically, the $\alpha$-mollified value determines the payoff allocation in two steps. First, an initial share of the worth of the grand coalition is determined by a linear function with respect to the worths of all coalitions. Second, this initial share is allocated over the players in proportion to their stand-alone worth, and the residual of the worth of the grand coalition

\footnotetext{
${ }^{1}$ The equal division value and the proportional division value are, respectively, called the equal sharing rule and the proportional sharing rule in Moulin (1987).
} 
is split equally over all players. This value reduces to the equal or proportional division value when the initial share is zero or the worth of the grand coalition, respectively. After introducing these values, we first provide an axiomatization of the family of $\alpha$-mollified values, as well as for affine combinations of the equal division value and the equal surplus division value (Driessen and Funaki, 1991).

Our second result identifies which members of our family satisfy projection consistency (see, e.g., Funaki and Yamato (2001); van den Brink and Funaki (2009); van den Brink et al. (2016) and Calleja and Llerena (2019)). The consistency principle has been successfully applied to characterize a wide variety of value concepts for TU-games. Given a payoff vector for some initial game, and given a subgroup of players, a so-called reduced game among these players is constructed where the worth of a coalition of remaining players depends on what they can earn with leaving players, but also taking account of the payoffs assigned to the leaving players. A value is consistent if it selects the same payoff allocation for any reduced game as for the initial game. Different values satisfy different reduced game consistency properties, where the difference is with respect to the way the reduced game is defined. In the literature, various consistency properties are applied, using different reduced games, which together with some properties characterize a unique point-valued or set-valued solution. Some of the contributions on various solutions can be found in, e.g., Hart and Mas-Colell (1989); van den Brink and Funaki (2009); van den Brink et al. (2016); Calleja and Llerena (2017, 2019), and the surveys of Driessen (1991) and Thomson (2011). ${ }^{2}$

Instead of characterizing a unique value among all values, in this paper we use consistency to identify a subclass of values from our class of $\alpha$-mollified values. More precisely, we focus on projection consistency exerting on a generalization of the $\alpha$-mollified values in which the initial share is measured as a general (possibly asymmetric) linear function with respect to the worths of all coalitions. As it turns out, projection consistency singles out either the proportional division value, or an egalitarian value (being affine combination of the equal division value and the equal surplus division value). This result, obtained from our novel analytical approach, provides an advantage of the proportional division value and egalitarian values above other $\alpha$-mollified (generalized) values. That is, through the

\footnotetext{
${ }^{2}$ In contrast to the remarkable research on characterizing values, there are relatively few, yet significant works on constructing consistent extensions of two-claimant rules, in the context of claims problems. Such works mainly focus on identifying which members of the two-claimant family can be generalized to general populations by requiring consistency; we refer to Thomson (2008, 2013, 2015b, 2019).
} 
$\alpha$-mollified values depending on all coalition worths, projection consistency just singles out these special cases.

Besides an axiomatic approach, we also provide a procedural implementation of the $\alpha$ mollified values by designing a dynamic allocation process based on a one-by-one formation of the grand coalition, which is similar to that of the weighted ENSC value in Hou et al. (2019).

This paper is organized as follows. Section 2 provides basic definitions and notation. Section 3 introduces the concept of the $\alpha$-mollified value. Section 4 provides an axiomatzation of the class of $\alpha$-mollified values. In Section 5, we focus on projection consistency exerting on our family. Section 6 gives a procedural implementation. Section 7 concludes. The proofs are provided in Section 8.

\section{Basic definitions and notation}

Let $N=\{1,2, \ldots, n\}$ be a finite set of players and $2^{N}=\{S \mid S \subseteq N\}$ be the set of its coalitions. For each coalition $S \subseteq N$, its cardinality will be denoted by $|S|$ or $s$. A cooperative game with transferable utility, shortly a TU-game or a game, is a pair $(N, v)$ where $N$ is a set of players and $v: 2^{N} \rightarrow \mathbb{R}$ is a characteristic function that assigns a real number $v(S)$ to each coalition $S \subseteq N$, satisfying $v(\emptyset)=0$. The class of games with player set $N$ is denoted by $\mathcal{G}^{N}$. We denote the set of positive real numbers by $\mathbb{R}_{+}$.

Following Béal et al. (2018), a game $(N, v)$ is individually positive if $v(\{i\})>0$ for all $i \in N$, and individually negative if $v(\{i\})<0$ for all $i \in N$. Béal et al. (2018) also provide many applications of this restrictive class of TU-games. Let $\mathcal{G}_{n z+}^{N}$ and $\mathcal{G}_{n z-}^{N}$ denote the classes of individually positive and individually negative games on a given player set $N$, respectively. We denote by $\mathcal{G}_{n z}^{N}$ the union of $\mathcal{G}_{n z+}^{N}$ and $\mathcal{G}_{n z-}^{N}$, and by $\mathcal{G}_{n z}$ the class of such games with at least two players, i.e. $\mathcal{G}_{n z}=\{(N, v)|| N \mid \geq 2$ and $[v(\{i\})>0$ for all $i \in$ $N$, or $v(\{i\})<0$ for all $i \in N]\}$. Besides, $\mathcal{G}_{D}^{N}$ denotes the class $\left\{(N, v) \in \mathcal{G}_{n z}^{N} \mid v(\{i\}) \neq\right.$ $v(\{j\})$ for some $i, j \in N\}$.

Given $(N, v),(N, w) \in \mathcal{G}^{N}$ and $a, b \in \mathbb{R}$, the game $(N, a v+b w) \in \mathcal{G}^{N}$ is defined by $(a v+b w)(S)=a v(S)+b w(S)$ for all $S \subseteq N$. A permutation of $N$ is a bijection $\pi: N \rightarrow N$ where $\pi(i)=k$ indicates that player $i$ has the $k$ th position. We denote $\Pi(N)$ as the set of all the $n$ ! permutations of $N$. For a game $(N, v) \in \mathcal{G}^{N}$ and permutation $\pi \in \Pi(N)$, the game $(N, \pi v)$ is given by $\pi v(S)=v(\{\pi(i) \mid \pi(i) \in S\})$ for all $S \subseteq N$. 
A (point-valued) solution or a value on a class of TU-games $\mathcal{C} \subseteq \mathcal{G}_{n z}$ is a function $\psi$ that assigns a single payoff vector $\psi(N, v) \in \mathbb{R}^{N}$ to every game $(N, v) \in \mathcal{C}$. We recall some well-known values.

The equal division (ED) value on $\mathcal{C} \subseteq \mathcal{G}_{n z}$ is defined for all $(N, v) \in \mathcal{C}$ and $i \in N$ by

$$
E D_{i}(N, v)=\frac{1}{n} v(N)
$$

The equal surplus division (ESD) value (also known as Center-of-the-imputation-set $(C I S)$-value, see Driessen and Funaki (1991)) on $\mathcal{C} \subseteq \mathcal{G}_{n z}$ is defined for all $(N, v) \in \mathcal{C}$ and $i \in N$ by

$$
E S D_{i}(N, v)=v(\{i\})+\frac{1}{n}\left[v(N)-\sum_{j \in N} v(\{j\})\right] .
$$

The proportional division $(P D)$ value on $\mathcal{C} \subseteq \mathcal{G}_{n z}$ is defined for all $(N, v) \in \mathcal{C}$ and $i \in N$ by

$$
P D_{i}(N, v)=\frac{v(\{i\})}{\sum_{k \in N} v(\{k\})} v(N) .
$$

For any real number $\beta \in[0,1]$, the convex combination of the $\mathrm{ED}$ value and PD value with respect to $\beta$, introduced by Moulin (1987) for a special type of game, is defined for all $(N, v) \in \mathcal{C}$ and $i \in N$ by

$$
\psi_{i}^{\beta}(N, v)=\beta P D_{i}(N, v)+(1-\beta) E D_{i}(N, v)
$$

We state some axioms of values for games.

- Efficiency. For all $(N, v) \in \mathcal{C} \subseteq \mathcal{G}_{n z}$, it holds that $\sum_{i \in N} \psi_{i}(N, v)=v(N)$.

- Linearity. For all $(N, v),(N, w) \in \mathcal{C} \subseteq \mathcal{G}_{n z}$ and $a, b \in \mathbb{R}$ such that $(N, a v+b w) \in \mathcal{C}$, it holds that $\psi(N, a v+b w)=a \psi(N, v)+b \psi(N, w)$.

- Anonymity. For all $(N, v) \in \mathcal{C} \subseteq \mathcal{G}_{n z}$, all permutation $\pi: N \rightarrow N$ and all $i \in N$ such that $(N, \pi v) \in \mathcal{C}$, it holds that $\psi_{i}(N, v)=\psi_{\pi(i)}(N, \pi v)$.

- Continuity. For all sequences of games $\left\{\left(N, w_{k}\right)\right\}$ of elements of $\mathcal{C} \subseteq \mathcal{G}_{n z}$ and every game $(N, v) \in \mathcal{C}$ such that $\left(N, w_{k}\right) \rightarrow(N, v)$, it holds that $\lim _{k \rightarrow \infty} \psi\left(N, w_{k}\right)=\psi(N, v)$.

- Weak additivity. For all $(N, v),(N, w) \in \mathcal{C} \subseteq \mathcal{G}_{n z}$ such that there exists $c \in \mathbb{R}$ with $w(\{i\})=c v(\{i\})$ for all $i \in N$, if $(N, v+w) \in \mathcal{C}$ then $\psi(N, v+w)=\psi(N, v)+\psi(N, w)$. 
Efficiency, linearity and anonymity are well-known. Continuity states that small changes in the parameters of the game do not bring big jumps in the payoffs. Weak additivity, proposed by Besner (2019), states that when taking the sum of two games, where the ratio between the stand-alone worths is the same in both games, the payoff vector equals the sum of the payoff vectors of the two separate games. This axiom weakens the weak linearity axiom in Béal et al. (2018) as well as the usual additivity.

\section{Compromising between the proportional and equal division values}

We generalize Formula (1) for TU-games by defining a new value, called $\alpha$-mollified value, that not only adopts the proportional and equal division principles, but also takes into account the worths of all coalitions.

Definition 1. Take a collection of parameters $\alpha_{s}^{n} \in \mathbb{R}$ for any $n \in \mathbb{N}$ and $s \leq n$. Let $\alpha(N, v)=\sum_{S \subseteq N} \alpha_{|S|}^{|N|} v(S)$ be a linear function depending on the numbers $\alpha_{|S|}^{|N|} \in \mathbb{R}$ and $(N, v)$. The $\alpha$-mollified value on $\mathcal{C} \subseteq \mathcal{G}_{n z}$ is defined for all $(N, v) \in \mathcal{C}$ and $i \in N$, by

$$
\psi_{i}^{\alpha(N, v)}(N, v)=\frac{v(\{i\})}{\sum_{k \in N} v(\{k\})} \alpha(N, v)+\frac{1}{n}(v(N)-\alpha(N, v)) .
$$

Dutta and Ray (1989) argue that all coalitions should be considered when formulating an (egalitarian) allocation in a TU-game. This is clearly not the case when one considers the equal or proportional division value, or any convex combination of them. However, since the linear function $\alpha$ used to define an $\alpha$-mollified value depends on all coalition worths, the payoff allocation according to an $\alpha$-mollified value might depend on all coalition worths. The idea of assigning numbers to TU-games, as done by the linear function $\alpha$ is similar to Hart and Mas-Colell (1989) who associate a real number with a TU-game, called potential, that is the expected normalized worth, being a linear function with respect to the worths of all coalitions. Casajus (2014) shows that the potential is the expected worth generated by some natural random partition of the player set.

If there is no ambiguity, we use $\psi^{\alpha}(N, v)$ instead of $\psi^{\alpha(N, v)}(N, v)$.

Remark 1. Various functions $\alpha(N, v)$ give rise to various values. Some examples are the following: 
(i) The ED value is obtained when $\alpha(N, v)=0$.

(ii) The ESD value is obtained when $\alpha(N, v)=\sum_{k \in N} v(\{k\})$.

(iii) The $\mathrm{PD}$ value is obtained when $\alpha(N, v)=v(N)$.

(iv) The family of affine combinations of the $\mathrm{ESD}$ value and $\mathrm{ED}$ value, i.e. $\psi=\beta E S D+$ $(1-\beta) E D$, is obtained when $\alpha(N, v)=\beta \sum_{k \in N} v(\{k\}), \beta \in \mathbb{R}$.

(v) The family of affine combinations of the $\mathrm{PD}$ value and $\mathrm{ED}$ value, i.e. $\psi=\beta P D+$ $(1-\beta) E D$, is obtained when $\alpha(N, v)=\beta v(N), \beta \in \mathbb{R}$.

(vi) The family of affine combinations of the $\mathrm{PD}$ value and $\mathrm{ESD}$ value, i.e. $\psi=\beta P D+$ $(1-\beta) E S D$, is obtained when $\alpha(N, v)=\beta v(N)+(1-\beta) \sum_{k \in N} v(\{k\}), \beta \in \mathbb{R}$.

(vii) The egalitarian proportional surplus division value (Zou et al., 2020) given by

$$
\operatorname{EPSD}_{i}(N, v)=\frac{1}{n} \sum_{k \in N} v(\{k\})+\frac{v(\{i\})}{\sum_{k \in N} v(\{k\})}\left[v(N)-\sum_{k \in N} v(\{k\})\right]
$$

is obtained when $\alpha(N, v)=v(N)-\sum_{k \in N} v(\{k\})$.

\section{Axiomatization of the family of $\alpha$-mollified values}

There are several approaches to justify values for TU-games. Two of these approaches are axiomatization and providing a dynamic process. In this section, we provide an axiomatization for the family of $\alpha$-mollified values, as well as for affine combinations of the ESD and ED values.

In order to characterize the family of $\alpha$-mollified values, we consider the following axioms. First, the balanced loss property states that the ratio of the difference of the payoffs of any two players over the difference of their stand-alone worths is equal for any pair of players. To avoid dividing by zero, we formulate this axiom as follows.

- Balanced loss property. For any $(N, v) \in \mathcal{G}_{n z}$ with $N \geq 3$ and any $i, j, k \in N$,

$$
\left(\psi_{i}(N, v)-\psi_{j}(N, v)\right)(v(\{i\})-v(\{k\}))=\left(\psi_{i}(N, v)-\psi_{k}(N, v)\right)(v(\{i\})-v(\{j\})) .
$$


For any game $(N, v) \in \mathcal{G}_{D}^{N}$, and players $i, j, h \in N$ with $v(\{i\}) \neq v(\{j\})$ and $v(\{i\}) \neq$ $v(\{k\}),(3)$ can be written as

$$
\frac{\psi_{i}(N, v)-\psi_{j}(N, v)}{v(\{i\})-v(\{j\})}=\frac{\psi_{i}(N, v)-\psi_{k}(N, v)}{v(\{i\})-v(\{k\})},
$$

where the denominators are nonzero for all $(N, v) \in \mathcal{G}_{D}^{N}$.

Under efficiency and continuity, the balanced loss property characterizes the following family of values.

Proposition 1. Let $|N| \geq 3$. A value $\psi$ on $\mathcal{G}_{n z}^{N}$ satisfies efficiency, the balanced loss property, and continuity if and only if there exists a continuous function $g: \mathcal{G}_{n z}^{N} \rightarrow \mathbb{R}$ such that

$$
\psi_{i}(N, v)=\frac{v(N)}{n}+g(N, v)\left[v(\{i\})-\frac{1}{n} \sum_{k \in N} v(\{k\})\right]
$$

for $\operatorname{all}(N, v) \in \mathcal{G}_{n z}^{N}$ and $i \in N$.

The proof of Proposition 1 and of all other results can be found in Section 8.

Remark 2. Proposition 1 still holds if the domain $\mathcal{G}_{n z}^{N}$ is replaced by $\mathcal{G}_{n z+}^{N}$ or $\mathcal{G}_{n z-}^{N}$, as follows from the proof of this proposition.

Among the values characterized in Proposition 1, only affine combinations of the ESD value and the ED value satisfy the linearity axiom. As shown below, continuity is superfluous in this characterization result.

Theorem 1. Let $|N| \geq 3$. A value $\psi$ on $\mathcal{G}_{n z}^{N}$ satisfies efficiency, the balanced loss property, and linearity if and only if there is $\beta \in \mathbb{R}$ such that

$$
\psi_{i}(N, v)=\beta v(\{i\})+\frac{1}{n}\left[v(N)-\sum_{k \in N} \beta v(\{k\})\right],
$$

for $\operatorname{all}(N, v) \in \mathcal{G}_{n z}^{N}$ and $i \in N$.

To characterize the family of $\alpha$-mollified values on $\mathcal{G}_{n z+}^{N}$ or $\mathcal{G}_{n z-}^{N}$, we add the following axiom. No advantageous reallocation across individuals (Moulin, 1987) states that no group of players benefits if reallocating their stand-alone worths among themselves is allowed. All efficient linear and symmetric values satisfy this axiom. Making use of a similar axiom, Ertemel and Kumar (2018) characterize an extension of the proportional rule for rationing problems, which is similar to our $\alpha$-mollified value in that context. 
- No advantageous reallocation across individuals. For all $(N, v),(N, w) \in$ $\mathcal{C} \subseteq \mathcal{G}_{n z}$ and $T \subseteq N$ such that (i) $v(S)=w(S)$ for all $S \subseteq N$ with $|S| \geq 2$, (ii) $v(\{i\})=w(\{i\})$ for all $i \in N \backslash T$, and (iii) $\sum_{i \in T} v(\{i\})=\sum_{i \in T} w(\{i\})$, it holds that $\sum_{i \in T} \psi_{i}(N, v)=\sum_{i \in T} \psi_{i}(N, w)$.

This axiom characterizes the family of $\alpha$-mollified values together with weak additivity, anonymity, and the axioms in Proposition 1.

Theorem 2. Let $|N| \geq 3$. A value $\psi$ on $\mathcal{G}_{n z+}^{N}$ (respectively $\mathcal{G}_{n z-}^{N}$ ) satisfies efficiency, the balanced loss property, continuity, weak additivity, anonymity, and no advantageous reallocation across individuals if and only if $\psi$ belongs to the family of $\alpha$-mollified values.

Again, the proof can be found in Section 8.

Remark 3. If weak additivity is replaced by weak linearity, then the coefficient $\alpha_{|S|}^{|N|}$ for each $S \subseteq N$ in (2) must be the same on $\mathcal{G}_{n z+}^{N}$ and $\mathcal{G}_{n z-}^{N}$, and thus in this case the domain in Theorem 2 can be extended to the class $\mathcal{G}_{n z}^{N}$. Together with Theorem 1 , we conclude that the affine combinations of the ESD value and the ED value are the only linear values in the family of $\alpha$-mollified values on $\mathcal{G}_{n z}^{N}$.

Logical independence of the axioms in Theorem 2 can be shown by the following alternative values on $\mathcal{G}_{n z+}^{N}\left(\right.$ or $\left.\mathcal{G}_{n z-}^{N}\right)$ :

(i) The value defined by $\psi_{i}(N, v)=0$ for all $i \in N$ satisfies all axioms except efficiency.

(ii) The value defined by

$$
\psi_{i}(N, v)=\frac{v(N)}{n}+\sum_{S \subseteq N: i \in S,|S| \geq 2} v(S)-\frac{1}{n} \sum_{S \subseteq N:|S| \geq 2}|S| v(S), \quad \text { for all } i \in N,
$$

satisfies all axioms except the balanced loss property.

(iii) Let $\widehat{f}: \mathbb{R}^{2^{n}-n-1} \rightarrow \mathbb{R}$ be a discontinuous additive function ${ }^{3}$ with respect to all $v(S)$, $S \subseteq N,|S| \geq 2$. The value defined by

$$
\psi_{i}(N, v)=\frac{v(N)}{n}+\widehat{f}(N, v)\left(\frac{v(\{i\})}{\sum_{k \in N} v(\{k\})}-\frac{1}{n}\right), \text { for all } i \in N,
$$

\footnotetext{
${ }^{3}$ The existence of discontinuous additive functions was an open problem for many years. Mathematicians could neither prove that every additive function is continuous nor exhibit an example of a discontinuous additive function. Hamel (1905) first succeeded in proving that there exist discontinuous additive functions. No concrete example is known, but we only know that it exists; we refer to (pp.129-130, Kuczma (2009)) and (pp.9-13, Sahoo and Kannappan (2011)).
} 
satisfies all axioms except continuity.

(iv) The value defined by

$$
\psi_{i}(N, v)=\frac{v(N)}{n}+\frac{v(\{i\})}{\sum_{k \in N} v(\{k\})}-\frac{1}{n}, \text { for all } i \in N,
$$

satisfies all axioms except weak additivity.

(v) Let $\left\{\alpha_{S}^{N}|S \subseteq N| S \mid, \geq 2\right\}$ be a collection of real numbers such that $\alpha_{T}^{N} \neq \alpha_{K}^{N}$ for some $T, K \subseteq N$ with $|T|=|K|$. The value defined by

$$
\psi_{i}(N, v)=\frac{v(N)}{n}+\sum_{S \subseteq N:|S| \geq 2} \alpha_{S}^{N} v(S)\left(\frac{v(\{i\})}{\sum_{k \in N} v(\{k\})}-\frac{1}{n}\right) \text {, for all } i \in N,
$$

satisfies all axioms except anonymity.

(vi) The value defined by

$$
\psi_{i}(N, v)=\frac{v(N)}{n}+\frac{\sum_{k \in N}(v(\{k\}))^{2}}{\sum_{k \in N} v(\{k\})}\left(\frac{v(\{i\})}{\sum_{k \in N} v(\{k\})}-\frac{1}{n}\right), \text { for all } i \in N \text {, }
$$

satisfies all axioms except no advantageous reallocation across individuals.

\section{Consistency}

It is shown that the affine combinations of the ED and ESD values can be characterized using projection consistency by van den Brink et al. (2016). Zou et al. (2019) use projection consistency to characterize the PD value. Several values satisfy projection consistency, see, e.g., Funaki and Yamato (2001); Calleja and Llerena (2017, 2019). However, we show that the only $\alpha$-mollified values that satisfy projection consistency are the PD value and affine combinations of the ED and ESD values.

We recall the definitions of the projection reduced game and projection consistency used in van den Brink and Funaki (2009) and van den Brink et al. (2016). If a player $j \in N$ leaves game $(N, v)$ with a certain payoff, then the projection reduced game is a game on the remaining player set that assigns to coalition $N \backslash\{j\}$ its worth in $(N, v)$ minus the

payoff assigned to player $j$, and assigns to every proper subset of $N \backslash\{j\}$ its worth in the original game. 
Definition 2. Given a game $(N, v) \in \mathcal{G}_{n z}$ with $|N| \geq 2$, a player $j \in N$ and a payoff vector $x \in \mathbb{R}^{N}$, the projection reduced game with respect to $j$ and $x$ is the game $\left(N \backslash\{j\}, v^{x}\right)$ given by

$$
v^{x}(S)= \begin{cases}v(N)-x_{j} & \text { if } S=N \backslash\{j\}, \\ v(S) & \text { if } S \subset N \backslash\{j\} .\end{cases}
$$

Projection consistency requires that the payoffs assigned to the remaining players in $N \backslash\{j\}$ in the reduced game, after player $j$ leaving the game with its payoff according to a value $\psi$, is the same as in the original game.

Definition 3. A value $\psi$ satisfies projection consistency if for every game $(N, v) \in \mathcal{G}_{n z}$ with $|N| \geq 3, j \in N$, and $x=\psi(N, v)$, it holds that $\left(N \backslash\{j\}, v^{x}\right) \in \mathcal{G}_{n z}$, and $\psi_{i}\left(N \backslash\{j\}, v^{x}\right)=$ $\psi_{i}(N, v)$ for all $i \in N \backslash\{j\}$.

Consistency is usually applied together with some properties to characterize a unique value on a class of games. Instead, the following theorem uses the consistency principle to select specific values from the family of $\alpha$-mollified values (and thus implicitly assumes properties that characterize this family in Theorem 2).

Theorem 3. Let $\psi^{\alpha}$ on $\mathcal{G}_{n z}$ be an $\alpha$-mollified value. Then $\psi^{\alpha}$ satisfies projection consistency if and only if $\psi^{\alpha}=P D$ or $\psi^{\alpha}=\beta E S D+(1-\beta) E D$, where $\beta \in \mathbb{R}$.

Although we can give a direct proof of this theorem, it follows as a corollary from the following theorem using a class of values containing the $\alpha$-mollified values. The difference is that in defining the linear functions $\alpha(N, v)$, we allow that coalitions of the same size are assigned a different weight by the function $\alpha(N, v)$.

Definition 4. Take a collection of parameters $\alpha_{S}^{N}$ for any $N$ and $S \subseteq N$. Let $\alpha(N, v)=$ $\sum_{S \subseteq N} \alpha_{S}^{N} v(S)$ be a linear function depending on $\alpha_{S}^{N}$ and $(N, v)$. The $\alpha$-mollified generalized value on $\mathcal{C} \subseteq \mathcal{G}_{n z}$ is defined for all $(N, v) \in \mathcal{C}$ and $i \in N$, by

$$
\psi_{i}^{\alpha(N, v)}(N, v)=\frac{v(\{i\})}{\sum_{k \in N} v(\{k\})} \alpha(N, v)+\frac{1}{n}(v(N)-\alpha(N, v)) .
$$

Notice that Expression (6) is the same as Expression (2), but we apply a more general function $\alpha(N, v)$. It turns out that Theorem 3 holds on the larger class of $\alpha$-mollified generalized values. 
Theorem 4. Let $\psi^{\alpha}$ on $\mathcal{G}_{n z}$ be an $\alpha$-mollified generalized value. Then $\psi^{\alpha}$ satisfies projection consistency if and only if $\psi^{\alpha}=P D$ or $\psi^{\alpha}=\beta E S D+(1-\beta) E D$, where $\beta \in \mathbb{R}$.

As mentioned before, the family of $\alpha$-mollified values is a subfamily of $\alpha$-mollified generalized values. Since the resulting values in Theorem 4 are also members in the family of $\alpha$-mollified values, we obtain Theorem 3 as a corollary of Theorem 4 .

As an implication of Theorem 4, the combinations of the PD value and any value belonging to $\{\gamma E S D+(1-\gamma) E D \mid \gamma \in \mathbb{R}\}$, such as the value given by (1) with $\beta \in(0,1)$, cannot be characterized using projection consistency.

\section{Procedural implementation}

As mentioned in Section 4, a dynamic process of allocating the attainable worth is another approach to justify values for TU-games; we refer to Hwang et al. (2005); Malawski (2013); Wang et al. (2019) and Hou et al. (2019). Under the assumption that all players form the grand coalition, as is usual in the theory of TU-games, the players then totally allocate the worth of the grand coalition among themselves. Given a formation order, a player claims his contribution to the grand coalition when he joins the game, and what's left is allocated among the players who have arrived before him. Motivated by this procedure, we give a procedural implementation of the $\alpha$-mollified values based on a one-by-one formation of the grand coalition.

Formally, a unique payoff vector is determined by the following steps:

Step 1: Choose any game $(N, v) \in \mathcal{G}_{n z}^{N}$ and any permutation $\pi \in \Pi(N)$ to gradually form the grand coalition $N$.

Step 2: Each entering player $i \in N$ such that $\pi(i)=1$ receives his individual worth $v(\{i\})$.

Step 3: Each entering player $i \in N$ such that $\pi(i) \neq 1$ obtains the fraction $\frac{v(\{i\})}{\sum_{k \in N} v(\{k\})}$ from the preservated worth $\alpha(N, v)$.

Step 4: The residual $v\left(S_{\pi}^{i}\right)-v\left(S_{\pi}^{i} \backslash\{i\}\right)-\frac{v(\{i\})}{\sum_{k \in N} v(\{k\})} \alpha(N, v)$ after player $i$ joining the nonempty coalition $S_{\pi}^{i} \backslash\{i\}, S_{\pi}^{i}=\{j \in N \mid \pi(j) \leq \pi(i)\}$, is allocated equally among the members of the coalition that was present before $i$ entered. 
Steps 1-4 determine a payoff vector $\left(\eta^{\pi, \alpha}\right)_{i \in N} \in \mathbb{R}^{N}$ defined as:

$$
\eta_{i}^{\pi, \alpha}= \begin{cases}v(\{i\})+\sum_{\substack{j \in N: \\ \pi(j)>\pi(i)}} \frac{v\left(S_{\pi}^{j}\right)-v\left(S_{\pi}^{j} \backslash\{j\}\right)-\frac{v(\{j\}) \alpha(N, v)}{\left.\left.\sum_{k \in N} v(k\}\right\}\right)}}{\pi(j)-1}, & \text { if } \pi(i)=1, \\ \frac{v(\{i\}) \alpha(N, v)}{\sum_{k \in N} v(\{k\})}+\sum_{\substack{j \in N: \\ \pi(j)>\pi(i)}} \frac{v\left(S_{\pi}^{j}\right)-v\left(S_{\pi}^{j} \backslash\{j\}\right)-\frac{v(\{j\}) \alpha(N, v)}{\sum_{k \in N} v(\{k\})}}{\pi(j)-1}, & \text { if } \pi(i) \neq 1, n, \\ \frac{v(\{i\}) \alpha(N, v)}{\sum_{k \in N} v(\{k\})}, & \text { if } \pi(i)=n .\end{cases}
$$

The next theorem shows that averaging the outcome of this procedure over all permutations yields the corresponding $\alpha$-mollified value. In fact, by the same proof it can be shown that this holds for any $\alpha$-mollified generalized value.

Theorem 5. For any $(N, v) \in \mathcal{G}_{n z}^{N}$ and any linear function $\alpha(N, v)=\sum_{S \subseteq N} \alpha_{|S|}^{|N|} v(S)$,

$$
\psi_{i}^{\alpha}(N, v)=\frac{1}{n !} \sum_{\pi \in \Pi(N)} \eta_{i}^{\pi, \alpha}, \quad \text { for all } i \in N
$$

where $\eta_{i}^{\pi, \alpha}$ is given by ( 7$)$.

Again, the proof can be found in Section 8.

Remark 4. Theorem 5 is still valid for games in which the sum of all stand-alone worths is nonzero. For the procedural implementation, the number $\frac{v(\{i\})}{\sum_{k \in N} v(\{k\})}$ for each $i \in N$ can be considered as an endogenous weight of player $i$. If this endogenous weight vector is replaced by an exogenous weight vector $\left(w_{i}\right)_{i \in N}$, i.e. $w_{i} \geq 0$ for all $i \in N$ and $\sum_{k \in N} w_{k}=1$, then the expected payoff is given by

$$
\psi_{i}^{w}(N, v)=w_{i} \alpha(N, v)+\frac{1}{n}(v(N)-\alpha(N, v)), \text { for all } i \in N .
$$

\section{Conclusion}

In this paper, we have introduced a parametric family of values, called $\alpha$-mollified values, that allocate the worth of the grand coalition based on proportional and equal division methods. We have axiomatized this family by employing efficiency, the balanced loss property, continuity, weak additivity, anonymity, and no advantageous reallocation across individuals. Moreover, we have provided a novel analytical approach to select the proportional division value and the affine combinations of the equal division value and the equal 
surplus division value from the class of $\alpha$-mollified values by imposing projection consistency. We did this using a larger class of $\alpha$-mollified generalized values. Finally, we have implemented each member of this family based on a one-by-one formation of the grand coalition.

Proportional and equal division are two famous allocation principles in economics. Besides combining these two principles, $\alpha$-mollified values also allow that the part of the worth of the grand coalition to which we apply proportionality, respectively equality, depends on the worths of all coalitions. Whereas usual proportional and equal division solutions only take account of the worths of singletons and the grand coalition, the $\alpha$-mollified values might thus depend on the worths of all coalitions. ${ }^{4}$

\section{Proofs}

Let us denote $K(v)=\sum_{i \in N} v(\{i\})$ for any game $(N, v)$. If no ambiguity is possible, we use $K$ instead of $K(v)$.

Proof of Proposition 1. It is clear that the 'if' part is satisfied. To show the 'only if' part, suppose that $\psi$ is a value satisfying efficiency, the balanced loss property, and continuity. We distinguish the games of $\mathcal{G}_{n z}^{N}$ into two cases.

Case (i): $(N, v) \in \mathcal{G}_{D}^{N}$. Notice that there being some pair of players $i, j \in N$ with $v(\{i\}) \neq v(\{j\})$, implies that for any $i \in N$, there must be some player $j \in N$ such that $v(\{i\}) \neq v(\{j\})$. The balanced loss property implies that for all $k \in N \backslash\{i\}$,

$$
\left(\psi_{i}(N, v)-\psi_{j}(N, v)\right)(v(\{i\})-v(\{k\}))=\left(\psi_{i}(N, v)-\psi_{k}(N, v)\right)(v(\{i\})-v(\{j\})) .
$$

Denoting $g_{i}(N, v)=\frac{\psi_{i}(N, v)-\psi_{j}(N, v)}{v(\{i\})-v(\{j\})}$, the above equation can be written as

$$
\psi_{i}(N, v)-\psi_{k}(N, v)=g_{i}(N, v)[v(\{i\})-v(\{k\})] .
$$

Summing this equality over all $k \in N \backslash\{i\}$, yields $(n-1) \psi_{i}(N, v)-\sum_{k \in N \backslash\{i\}} \psi_{k}(N, v)=$ $g_{i}(N, v)\left[(n-1) v(\{i\})-\sum_{k \in N \backslash\{i\}} v(\{k\})\right]$, which can be rewritten as

$$
n \psi_{i}(N, v)-\sum_{k \in N} \psi_{k}(N, v)=g_{i}(N, v)\left[n v(\{i\})-\sum_{k \in N} v(\{k\})\right] .
$$

\footnotetext{
${ }^{4}$ Other proportional solutions that take the worths of all coalitions into account are the proper Shapley values (Vorobev and Liapunov, 1998) or the proportional Shapley value (Béal et al., 2018).
} 
With efficiency, $\sum_{k \in N} \psi_{k}(N, v)=v(N)$, and thus this implies

$$
\psi_{i}(N, v)=\frac{v(N)}{n}+g_{i}(N, v)\left[v(\{i\})-\frac{1}{n} \sum_{k \in N} v(\{k\})\right] .
$$

Notice that, for every player $i$ in a game $(N, v)$, the number $g_{i}(N, v)$ is determined for a specific $j$, but this $j$ can be different for different games (as long as it is a player with a different stand-alone worth as $i$.) Next, we show that $g_{i}(N, v)=g_{h}(N, v)$ for all $i, h \in N$. Let $i, h \in N$ be two players such that $v(\{i\}) \neq v(\{h\})$. Clearly, $g_{i}(N, v)=g_{h}(N, v)=$ $\frac{\psi_{i}(N, v)-\psi_{h}(N, v)}{v(\{i\})-v(\{h\})}$. For any $k \in N \backslash\{i, h\}$, it must be that $v(\{k\}) \neq v(\{i\})$ or $v(\{k\}) \neq$ $v(\{h\})$ (or both). Without loss of generality, we assume that $v(\{k\}) \neq v(\{i\})$. By the balanced loss property applied to $i, h, k \in N$, we have $\frac{\psi_{i}(N, v)-\psi_{h}(N, v)}{v(\{i\})-v(\{h\})}=\frac{\psi_{i}(N, v)-\psi_{k}(N, v)}{v(\{i\})-v(\{k\})}$, implying $g_{i}(N, v)=g_{k}(N, v)$.

Setting $g(N, v)=g_{i}(N, v)$ and then substituting it into (8), we obtain (4). Moreover, continuity implies that $g(N, v)$ is continuous.

Case (ii): $(N, v) \in \mathcal{G}_{n z}^{N} \backslash \mathcal{G}_{D}^{N}$. In this case, $v(\{i\})=v(\{j\})$ for all $i, j \in N$. Let $\left\{\left(N, w_{m}\right)\right\}$ be a sequence of games from $\mathcal{G}_{D}^{N}$ such that $\left(N, w_{m}\right) \rightarrow(N, v)$. By continuity and Case (i),

$$
\begin{aligned}
\psi_{i}(N, v) & =\lim _{m \rightarrow \infty} \psi_{i}\left(N, w_{m}\right) \\
& =\lim _{m \rightarrow \infty}\left[\frac{v(N)}{n}+g\left(N, w_{m}\right)\left[w_{m}(\{i\})-\frac{1}{n} \sum_{k \in N} w_{m}(\{k\})\right]\right] \\
& =\frac{v(N)}{n}+\lim _{m \rightarrow \infty} g\left(N, w_{m}\right)\left[w_{m}(\{i\})-\frac{1}{n} \sum_{k \in N} w_{m}(\{k\})\right] \\
& =\frac{v(N)}{n},
\end{aligned}
$$

where the last equality follows from the fact that $g\left(N, w_{m}\right)$ is a continuous function and, by $\left(N, w_{m}\right) \rightarrow(N, v)$ with $(N, v) \notin \mathcal{G}_{D}^{N}, \lim _{m \rightarrow \infty}\left[w_{m}(\{i\})-\frac{1}{n} \sum_{k \in N} w_{m}(\{k\})\right]=0$. Clearly, this coincides with (4), for any function $g(N, v)$. Taking $g(N, v)=\lim _{m \rightarrow \infty} g\left(N, w_{m}\right)$ yields the desired assertion.

Proof of Theorem 1. It is clear that any value of the form given in (5) satisfies the three axioms. To prove the 'only if ' part, suppose that $\psi$ is a value on $\mathcal{G}_{n z}^{N}$ satisfying the three axioms. The proof is divided into three steps. 
Step 1. We show a relationship among the payoffs of three games $(N, v),\left(N, v_{1}\right)$, $\left(N, v_{2}\right) \in \mathcal{G}_{D}^{N}$ such that $v=v_{1}+v_{2}$. From case (i) of the proof of Proposition 1, efficiency and the balanced loss property imply that $\psi$ on $\mathcal{G}_{D}^{N}$ has the form given in (4), but $g$ is not guaranteed to be continuous. By linearity, we have $\psi_{h}\left(N, v_{1}\right)+\psi_{h}\left(N, v_{2}\right)=\psi_{h}(N, v)$ for all $h \in N$. This yields

$$
\begin{aligned}
& g\left(N, v_{1}\right)\left[v_{1}(\{h\})-\frac{1}{n} K\left(v_{1}\right)\right]+g\left(N, v_{2}\right)\left[v_{2}(\{h\})-\frac{1}{n} K\left(v_{2}\right)\right] \\
= & g(N, v)\left[v(\{h\})-\frac{1}{n} K(v)\right] \\
= & g(N, v)\left[v_{1}(\{h\})+v_{2}(\{h\})-\frac{1}{n} K\left(v_{1}\right)-\frac{1}{n} K\left(v_{2}\right)\right] .
\end{aligned}
$$

Denote $x=g(N, v)-g\left(N, v_{1}\right)$ and $y=g(N, v)-g\left(N, v_{2}\right)$. The above equation can then be rewritten as

$$
x\left[v_{1}(\{h\})-\frac{1}{n} K\left(v_{1}\right)\right]+y\left[v_{2}(\{h\})-\frac{1}{n} K\left(v_{2}\right)\right]=0 .
$$

Subtracting these equations for any two distinct players $h, l \in N$ yields

$$
x\left[v_{1}(\{h\})-v_{1}(\{l\})\right]+y\left[v_{2}(\{h\})-v_{2}(\{l\})\right]=0 .
$$

Step 2. Next, using (9) we show that $g$ is a constant function on $\mathcal{G}_{D}^{N}$. We remark that we only have to consider the individually positive games, since $(N, v) \in \mathcal{G}_{D}^{N}$ implies $(N,-v) \in \mathcal{G}_{D}^{N}$. By (4) applied to $(N,-v)$, we have

$$
\begin{aligned}
\psi_{i}(N,-v) & =\frac{-v(N)}{n}+g(N,-v)\left[-v(\{i\})+\frac{1}{n} \sum_{k \in N} v(\{k\})\right] \\
& =-\left[\frac{v(N)}{n}+g(N,-v)\left[v(\{i\})-\frac{1}{n} \sum_{k \in N} v(\{k\})\right] .\right.
\end{aligned}
$$

By linearity, $\psi_{i}(N,-v)=-\psi_{i}(N, v)$. Taking into account the above equation and (4), we obtain $g(N, v)=g(N,-v)$.

Let $(N, v),(N, w) \in \mathcal{G}_{D}^{N} \cap \mathcal{G}_{n z+}^{N}$ and $i, j \in N$ be such that $v(\{i\}) \neq v(\{j\})$. Clearly, there must be a player $k \in N$ such that $w(\{k\}) \neq w(\{i\})$ or $w(\{k\}) \neq w(\{j\})$. Without loss of generality, we assume that $w(\{i\}) \neq w(\{k\})$ for a given $k \in N \backslash\{i, j\}$. To show that $g$ is a constant, we consider two cases:

Case (i): Suppose that $v(\{i\})-v(\{j\}) \neq w(\{i\})-w(\{j\})$ and $v(\{i\})-v(\{k\}) \neq$ $w(\{i\})-w(\{k\})$. Denote $\varepsilon=\sum_{h \in\{i, j, k\}}[v(\{h\})+w(\{h\})]$. We define the following three 
games.

$$
\begin{aligned}
& u(S)= \begin{cases}\varepsilon, & \text { if } S=\{i\}, \\
v(\{j\})-v(\{i\})+\varepsilon, & \text { if } S=\{j\}, \\
w(\{k\})-w(\{i\})+\varepsilon, & \text { if } S=\{k\}, \\
v(\{h\})+w(\{h\}), & \text { if } S=\{h\}, h \in N \backslash\{i, j, k\} . \\
0, & \text { if } S \subseteq N \text { with }|S| \geq 2 .\end{cases} \\
& v_{0}(S)= \begin{cases}v(\{i\})-\varepsilon, & \text { if } S=\{h\}, h \in\{i, j\}, \\
v(\{k\})-w(\{k\})+w(\{i\})-\varepsilon, & \text { if } S=\{k\}, \\
-w(\{h\}), & \text { if } S=\{h\}, h \in N \backslash\{i, j, k\} . \\
v(S), & \text { if } S \subseteq N \text { with }|S| \geq 2 .\end{cases} \\
& w_{0}(S)= \begin{cases}w(\{i\})-\varepsilon, & \text { if } S=\{h\}, h \in\{i, k\}, \\
w(\{j\})-v(\{j\})+v(\{i\})-\varepsilon, & \text { if } S=\{j\}, \\
-v(\{h\}), & \text { if } S=\{h\}, h \in N \backslash\{i, j, k\} . \\
w(S), & \text { if } S \subseteq N \text { with }|S| \geq 2 .\end{cases}
\end{aligned}
$$

Clearly, (i) $(N, u)$ is individually positive with $u(\{i\}) \neq u(\{j\})$ and $u(\{i\}) \neq u(\{k\})$; (ii) $\left(N, v_{0}\right)$ is individually negative with $v_{0}(\{i\}) \neq v_{0}(\{k\})$; (iii) $\left(N, w_{0}\right)$ is individually negative with $w_{0}(\{i\}) \neq w_{0}(\{j\})$. Moreover, $(N, u)+\left(N, v_{0}\right)=(N, v)$ and $(N, u)+$ $\left(N, w_{0}\right)=(N, w)$.

By (9) applied to $(N, u),\left(N, v_{0}\right),(N, v)$, and players $i, j$, we have

$$
x[u(\{i\})-u(\{j\})]+y\left[v_{0}(\{i\})-v_{0}(\{j\})\right]=0 .
$$

Since $u(\{i\})-u(\{j\}) \neq 0$ and $v_{0}(\{i\})-v_{0}(\{j\})=0$, then

$$
x=g(N, v)-g(N, u)=0 .
$$

Similarly, by $(9)$ applied to $(N, u),\left(N, w_{0}\right),(N, w)$, and players $i, k$, we have

$$
g(N, w)-g(N, u)=0 .
$$

Together, (10) and (11) imply

$$
g(N, v)=g(N, w)
$$


Case (ii): Suppose that $v(\{i\})-v(\{j\})=w(\{i\})-w(\{j\})$ or/and $v(\{i\})-v(\{k\})=$ $w(\{i\})-w(\{k\})$. Let $\varepsilon_{1}, \varepsilon_{2}, \varepsilon_{3} \in \mathbb{R}_{+}$be such that $\varepsilon_{3}<\min \{v(\{i\}), v(\{j\})\}+\varepsilon_{1}$. Consider the following games.

$$
\begin{aligned}
& v_{3}(S)= \begin{cases}v(S)+\varepsilon_{1}, & \text { if } S=\{h\}, h \in\{i, j\}, \\
v(S)+\varepsilon_{2}, & \text { if } S=\{h\}, h \in N \backslash\{i, j\} . \\
v(S), & \text { if } S \subseteq N \text { with }|S| \geq 2 .\end{cases} \\
& v_{4}(S)= \begin{cases}-\varepsilon_{1}, & \text { if } S=\{h\}, h \in\{i, j\}, \\
-\varepsilon_{2}, & \text { if } S=\{h\}, h \in N \backslash\{i, j\} . \\
0, & \text { if } S \subseteq N \text { with }|S| \geq 2 .\end{cases} \\
& v_{5}(S)= \begin{cases}v(S)+\varepsilon_{1}-\varepsilon_{3}, & \text { if } S=\{h\}, h \in\{i, j\}, \\
v(S), & \text { otherwise. }\end{cases} \\
& v_{6}(S)= \begin{cases}\varepsilon_{3}, & \text { if } S=\{h\}, h \in\{i, j\}, \\
\varepsilon_{2}, & \text { if } S=\{h\}, h \in N \backslash\{i, j\} . \\
0, & \text { if } S \subseteq N \text { with }|S| \geq 2 .\end{cases}
\end{aligned}
$$

Notice that $\left(N, v_{3}\right),\left(N, v_{5}\right)$ and $\left(N, v_{6}\right)$ are individually positive games, and $\left(N, v_{4}\right)$ is an individually negative game. Clearly, $(N, v)=\left(N, v_{3}\right)+\left(N, v_{4}\right)$ and $\left(N, v_{3}\right)=$ $\left(N, v_{5}\right)+\left(N, v_{6}\right)$. By $(9)$, we have $x\left(v_{3}(\{i\})-v_{3}(\{j\})\right)+y\left(v_{4}(\{i\})-v_{4}(\{j\})\right)=0$, and since $v_{3}(\{i\}) \neq v_{3}(\{j\})$ and $v_{4}(\{i\})=v_{4}(\{j\})$, we have $x=g(N, v)-g\left(N, v_{3}\right)=0$. Similar, $x\left(v_{5}(\{i\})-v_{5}(\{j\})\right)+y\left(v_{6}(\{i\})-v_{6}(\{j\})\right)=0$, and since $v_{5}(\{i\}) \neq v_{5}(\{j\})$ and $v_{6}(\{i\})=v_{6}(\{j\})$, we have $x=g\left(N, v_{3}\right)-g\left(N, v_{5}\right)=0$. Thus,

$$
g(N, v)=g\left(N, v_{5}\right)
$$

showing that $g$ remains unchanged if two different stand-alone worths change by the same amount, and the new game still is a member of $\mathcal{G}_{n z}^{N}$.

With (13), we can construct a game $\left(N, v^{\prime}\right) \in \mathcal{G}_{D}^{N}$ such that $g\left(N, v^{\prime}\right)=g(N, v), v^{\prime}(\{i\}) \neq$ $v^{\prime}(\{j\}), v^{\prime}(\{i\})-v^{\prime}(\{j\}) \neq w(\{i\})-w(\{j\})$ and $v^{\prime}(\{i\})-v^{\prime}(\{k\}) \neq w(\{i\})-w(\{k\}) .^{5}$ Since $\left(N, v^{\prime}\right)$ is as in Case (i), from Case (i) we have $g\left(N, v^{\prime}\right)=g(N, w)$. Thus, $g(N, v)=$ $g(N, w)$.

\footnotetext{
${ }^{5}$ To illustrate this for the case that $v(\{i\})-v(\{j\})=w(\{i\})-w(\{j\})$ and $v(\{i\})-v(\{k\})=$ $w(\{i\})-w(\{k\})$ (the case that only one of these equalities is satisfied goes similar), we can construct
} 
Cases (i) and (ii) show that $g(N, v)=g(N, w)$ for all $(N, v),(N, w) \in \mathcal{G}_{D}^{N}$, and thus $g$ is a constant function on $\mathcal{G}_{D}^{N}$. Setting $\beta=g(N, v)$, we obtain (5) on the class $\mathcal{G}_{D}^{N}$.

Step 3. Finally, we show that $g$ is a constant function on $\mathcal{G}_{n z}^{N}$, and thus (5) holds on $\mathcal{G}_{n z}^{N}$. From Step 2, we obtain (5) only on the class $\mathcal{G}_{D}^{N}$. On the other hand, consider any game $(N, v) \in \mathcal{G}_{n z}^{N}$ such that $v(\{i\})=v(\{j\})$ for all $i, j \in N$. Obviously, there exists two games $\left(N, v_{1}\right),\left(N, v_{2}\right) \in \mathcal{G}_{D}^{N}$ such that $(N, v)=\left(N, v_{1}\right)+\left(N, v_{2}\right)$. We obtain from Step 2 that $g\left(N, v_{1}\right)=g\left(N, v_{2}\right)=\beta$. By linearity and (4) applied to $\left(N, v_{1}\right)$ and $\left(N, v_{2}\right)$, we have

$$
\begin{aligned}
\psi_{i}(N, v)= & \psi_{i}\left(N, v_{1}\right)+\psi_{i}\left(N, v_{2}\right) \\
= & \frac{v_{1}(N)}{n}+\beta\left[v_{1}(\{i\})-\frac{1}{n} \sum_{k \in N} v_{1}(\{k\})\right] \\
& +\frac{v_{2}(N)}{n}+\beta\left[v_{2}(\{i\})-\frac{1}{n} \sum_{k \in N} v_{2}(\{k\})\right] \\
= & \frac{v_{1}(N)+v_{2}(N)}{n}+\beta\left[v_{1}(\{i\})+v_{2}(\{i\})-\frac{1}{n} \sum_{k \in N}\left(v_{1}(\{k\})+v_{2}(\{k\})\right)\right] \\
= & \frac{v(N)}{n}+\beta\left[v(\{i\})-\frac{1}{n} \sum_{k \in N} v(\{k\})\right] \\
= & \frac{v(N)}{n}
\end{aligned}
$$

where the last equality holds since $v(\{i\})=v(\{j\})$ for all $i, j \in N$. This is the ED value for any $\beta \in \mathbb{R}$, and coincides with (1) if $v(\{i\})=v(\{j\})$ for all $i, j \in N$.

\section{Proof of Theorem 2}

Before presenting the lengthy proof of Theorem 2, we introduce two theorems on Cauchy functional equations.

Theorem 6. (see, Theorem 5.5.2, p.139, Kuczma (2009)) If $f: \mathbb{R}^{N} \rightarrow \mathbb{R}$ is a continuous additive function, then there exists $d \in \mathbb{R}^{N}$ such that $f(x)=\sum_{i \in N} d_{i} x_{i}$.

the game $\left(N, v^{\prime}\right)$ given by $v^{\prime}(\{i\})=v(\{i\})+2 v(\{k\}), v^{\prime}(\{j\})=v(\{j\})+2 v(\{k\})+v(\{i\}), v^{\prime}(\{k\})=$ $v(\{k\})+v(\{i\})$ and $v^{\prime}(S)=v(S)$ otherwise. Obviously, $v^{\prime}(\{i\}) \neq v^{\prime}(\{j\}), v^{\prime}(\{i\})-v^{\prime}(\{j\})=-v(\{j\}) \neq$ $w(\{i\})-w(\{j\})$ and $v^{\prime}(\{i\})-v^{\prime}(\{k\})=v(\{k\}) \neq w(\{i\})-w(\{k\})$. Going from $(N, v)$ to $\left(N, v^{\prime}\right)$ in two steps $([v(\{i\}), v(\{j\}), v(\{k\})] \rightarrow[v(\{i\})+2 v(\{k\}), v(\{j\})+2 v(\{k\}), v(\{k\})] \rightarrow[v(\{i\})+2 v(\{k\})$, $v(\{j\})+2 v(\{k\})+v(\{i\}), v(\{k\})+v(\{i\})]$, we can twice apply that the value of function $g$ does not change, and thus $g(N, v)=g\left(N, v^{\prime}\right)$. 
For $x, y \in \mathbb{R}^{n}$, let $x-y=\left(x_{1}-y_{2}, x_{2}-y_{2}, \ldots, x_{n}-y_{n}\right)$.

Theorem 7. Let $f: \mathbb{R}_{+} \times \mathbb{R}^{n-1} \rightarrow \mathbb{R}$ be an additive function. Then $F: \mathbb{R}^{n} \rightarrow \mathbb{R}$ given by

$$
F(x-y)=f(x)-f(y), \quad \text { for all } x, y \in \mathbb{R}_{+} \times \mathbb{R}^{n-1}
$$

is an additive function such that $F(x)=f(x)$ for all $x \in \mathbb{R}_{+} \times \mathbb{R}^{n-1}$.

Proof. First, we show that $F$ is well-defined (that is, the function given in (14) is a valid definition of a function). Let $x, y, h, l \in \mathbb{R}_{+} \times \mathbb{R}^{n-1}$ be such that $x-h=y-l$. Hence, $x+l=y+h \Rightarrow f(x+l)=f(y+h) \Rightarrow f(x)+f(l)=f(y)+f(h) \Rightarrow f(x)-f(h)=f(y)-f(l)$ $\Rightarrow F(x-h)=F(y-l)$, where the second implication follow from additivity of $f$.

Next, we show that $F$ is an extension of $f$. For any $t \in \mathbb{R}_{+} \times \mathbb{R}^{n-1}$, there exist $x, y \in \mathbb{R}_{+} \times \mathbb{R}^{n-1}$ such that $t=x-y$. Hence, $F(t)=F(x-y)=f(x)-f(y)=$ $f(y+t)-f(y)=f(y)+f(t)-f(y)=f(t)$, as asserted.

Finally, we show that $F$ is additive on $\mathbb{R}^{n}$. For any $s, t \in \mathbb{R}^{n}$, there exist $x, y, h, l \in$ $\mathbb{R}_{+} \times \mathbb{R}^{n-1}$ such that $s=x-h$ and $t=y-l$. Note that $x+y$ and $t+l$ are in $\mathbb{R}_{+} \times \mathbb{R}^{n-1}$. Also, $s+t=(x+y)-(h+l)$. Then, by (14), we have $F(s+t)=F((x+y)-(h+l))=$ $f(x+y)-f(h+l)=f(x)+f(y)-f(h)-f(l)=F(x-h)+F(y-l)=F(s)+F(t)$, as asserted, where the third equality follows from additivity of $f$.

Theorem 7 is a modification of Theorem 2 in Aczél and Erdös (1965) that provides an extensive principle on conditional Cauchy equation ${ }^{6}$. A similar theorem (with a similar proof) holds if $\mathbb{R}_{+} \times \mathbb{R}^{n-1}$ is replaced by $\mathbb{R}_{-} \times \mathbb{R}^{n-1}$.

Next, we give additional notation and a remark. For any $c=\left(c_{i}\right)_{i \in N} \in \mathbb{R}_{+}^{N}$, the classes $\mathcal{G}_{c+}^{N}$ and $\mathcal{G}_{c-}^{N}$ are denoted as follows.

$$
\begin{aligned}
& \mathcal{G}_{c+}^{N}=\left\{(N, v) \in \mathcal{G}_{n z}^{N} \mid \exists a \in \mathbb{R}_{+}: \forall i \in N, v(\{i\})=a c_{i}\right\} . \\
& \mathcal{G}_{c-}^{N}=\left\{(N, v) \in \mathcal{G}_{n z}^{N} \mid \exists a \in \mathbb{R}_{-}: \forall i \in N, v(\{i\})=a c_{i}\right\} .
\end{aligned}
$$

That is, $\mathcal{G}_{c+}^{N}$ (respectively $\mathcal{G}_{c-}^{N}$ ) consists of all games in $\mathcal{G}_{n z}^{N}$ in which the players' stand-alone worths are in the same positive (respectively negative) proportion to $c$.

6 The Cauchy functional equation is a well-known and fundamental equation in the theory of functional equations. It is given by $f(x+y)=f(x)+f(y)$, where $f: \mathbb{R} \rightarrow \mathbb{R}$ is a continuous function. A conditional Cauchy equation is a variation of this equation by changing the domain of validity of the equation. 
Remark 5. Each game $(N, v) \in \mathcal{G}_{c+}^{N}$ is represented by a vector $p^{v}=\left(p_{1}^{v}, p_{S}^{v}\right) \in \mathbb{R}_{+} \times$ $\mathbb{R}^{2^{n}-n-1}$, where the first component $p_{1}^{v}=\frac{v(\{i\})}{c_{i}}$ is the ratio of $v(\{i\})$ to $c_{i}$ which is equal and positive for all $i \in N$, and the remaining $2^{n}-n-1$ components are the worths $v(S)$ of the $2^{n}-n-1$ coalitions $S \subseteq N,|S| \geq 2$. Moreover, $(N, v) \in \mathcal{G}_{c+}^{N} \Leftrightarrow p^{v} \in \mathbb{R}_{+} \times \mathbb{R}^{2^{n}-n-1}$. Similarly, $(N, v) \in \mathcal{G}_{c-}^{N} \Leftrightarrow p^{v} \in \mathbb{R}_{-} \times \mathbb{R}^{2^{n}-n-1}$. Clearly, $(N, v)=\left(N, v_{1}\right)+\left(N, v_{2}\right) \Leftrightarrow p^{v}=$ $p^{v_{1}}+p^{v_{2}}$.

Proof of Theorem 2. It is easily checked that any value of the form given in (2) satisfies the six axioms. To show the 'only if' part, let $\psi$ be a value on $\mathcal{G}_{n z+}^{N}$ satisfying the six axioms. (The proof on $\mathcal{G}_{n z-}^{N}$ goes in a similar way.) From Remark $2, \psi$ has the form given by (4). Define a function $f: \mathcal{G}_{n z+}^{N} \rightarrow \mathbb{R}$ by $f(N, v)=K(v) g(N, v)$ for all $(N, v) \in \mathcal{G}_{n z+}^{N}$, where $K(v)=\sum_{i \in N} v(\{i\})$, as defined in the beginning of Section 8. Then (4) can be rewritten as

$$
\psi_{i}(N, v)=\frac{v(N)}{n}+f(N, v)\left(\frac{v(\{i\})}{K(v)}-\frac{1}{n}\right) .
$$

Clearly, $f(N, v)$ is continuous since $K(v)$ and $g(N, v)$ are continuous.

We will consider five steps to show the rest of the 'only if' part. Step 1 formulates the value on $\mathcal{G}_{c+}^{N}$ that satisfies efficiency, the balanced loss property, continuity, and weak additivity. Using no advantageous reallocation across individuals, Step 2 and Step 3 derive the coefficients of the formula obtained in Step 1 if $c \in \mathbb{R}_{+}^{N}$ is such that $c_{i} \neq c_{j}$ for some $i, j \in N$. Step 4 considers the case that $c \in \mathbb{R}_{+}^{N}$ with $c_{i}=c_{j}$ for all $i, j \in N$. Step 5 gives the desired formula by anonymity.

Step 1. Pick any $c \in \mathbb{R}_{+}^{N}$. We derive the formula of $\psi$ on $\mathcal{G}_{c+}^{N}$ satisfying efficiency, the balanced loss property, continuity, and weak additivity. Consider $(N, v),(N, w) \in \mathcal{G}_{c+}^{N}$. Since $(N, v+w) \in \mathcal{G}_{c+}^{N}$, weak additivity implies that $\psi_{i}(N, v+w)=\psi_{i}(N, v)+\psi_{i}(N, w)$ for all $i \in N$. Taking (15) into account, we obtain

$$
f(N, v+w)=f(N, v)+f(N, w) .
$$

Let $h: \mathbb{R}_{+} \times \mathbb{R}^{2^{n}-n-1} \rightarrow \mathbb{R}$ be defined by $h\left(p^{v}\right)=f(N, v)$ for all $p^{v} \in \mathbb{R}_{+} \times \mathbb{R}^{2^{n}-n-1}$ with $(N, v) \in \mathcal{G}_{c+}^{N}$ as in Remark 5. Then, (16) can be rewritten as

$$
h\left(p^{v+w}\right)=h\left(p^{v}+p^{w}\right)=h\left(p^{v}\right)+h\left(p^{w}\right),
$$

which shows that $h$ is an additive function on $\mathbb{R}_{+} \times \mathbb{R}^{2^{n}-n-1}$. 
Moreover, $h$ is continuous since $f$ is continuous. Hence, from Theorem 7, there exists an additive function $H: \mathbb{R}^{2^{n}-n} \rightarrow \mathbb{R}$ such that $H(x)=h(x)$ for all $x \in \mathbb{R}_{+} \times \mathbb{R}^{2^{n}-n-1}$. Obviously, $H$ is continuous since (i) $H$ has the form of (14), i.e. $H(x-y)=h(x)-h(y)$ for all $x, y \in \mathbb{R}_{+} \times \mathbb{R}^{2^{n}-n-1}$, and (ii) $h$ is continuous. Thus, from Theorem 6 , there exists $d \in \mathbb{R}^{2^{n}-n}$ such that

$$
h(x)=H(x)=\sum_{i=1}^{2^{n}-n} d_{i} x_{i}, \quad \text { for all } x \in \mathbb{R}_{+} \times \mathbb{R}^{2^{n}-n-1} .
$$

Equivalently, by definition of $h$,

$$
f(N, v)=h\left(p^{v}\right)=d_{1}^{c} p_{1}^{v}+\sum_{S \subseteq N,|S| \geq 2} d_{S}^{c} v(S), \quad \text { for all }(N, v) \in \mathcal{G}_{c+}^{N},
$$

where $d_{1}^{c}$ and $d_{S}^{c}, S \subseteq N,|S| \geq 2$, are real numbers, and $p_{1}^{v}=\frac{v(\{i\})}{c_{i}} \in \mathbb{R}$ for all $i \in N$ is the ratio of $v(\{i\})$ to $c_{i}$. Clearly, these coefficients depend on $c$ since $(N, v) \in \mathcal{G}_{c+}^{N}$.

Taking into account that $d_{1}^{c}=d_{\{i\}}^{c} \in \mathbb{R}$ and $p_{1}^{v}=\frac{v(\{i\})}{c_{i}}$ for each $i \in N$, from (17), we obtain a system of $n$ linearly independent equations. Summing these equations over all players, we obtain

$$
n f(N, v)=\sum_{i \in N} \frac{d_{\{i\}}^{c}}{c_{i}} v(\{i\})+n \sum_{S \subseteq N,|S| \geq 2} d_{S}^{c} v(S) .
$$

Since $\mathcal{G}_{c+}^{N}=\mathcal{G}_{\beta c+}^{N}$ for all $\beta \in \mathbb{R}_{+}$, then $(N, v) \in \mathcal{G}_{c+}^{N}$ implies $(N, v) \in \mathcal{G}_{\beta c+}^{N}$. Thus, similar to (18), we have

$$
n f(N, v)=\sum_{i \in N} \frac{d_{\{i\}}^{\beta c}}{\beta c_{i}} v(\{i\})+n \sum_{S \subseteq N,|S| \geq 2} d_{S}^{\beta c} v(S) .
$$

Notice that the left-hand side of (18) is identical to that of (19), which yields

$$
\frac{d_{\{i\}}^{c}}{c_{i}}=\frac{d_{\{i\}}^{\beta c}}{\beta c_{i}}, \text { and } d_{S}^{c}=d_{S}^{\beta c} \text { for each } S \subseteq N,|S| \geq 2 .
$$

Therefore, setting $\alpha_{\{i\}}^{c}:=\frac{d_{\{i\}}^{c}}{n c_{i}}$ and $\alpha_{S}^{c}:=d_{S}^{c}$, (18) can be rewritten as

$$
f(N, v)=\sum_{S \subseteq N} \alpha_{S}^{c} v(S) .
$$

Notice that $\alpha_{S}^{c}$ is a real number depending on $S$ and $c$, and, moreover, for each $S \subseteq N$ with $|S| \geq 2$ and all $\beta \in \mathbb{R}_{+}$,

$$
\alpha_{S}^{c}=\alpha_{S}^{\beta c}
$$


Substituting (20) into (15), we obtain

$$
\psi_{i}(N, v)=\frac{v(N)}{n}+\sum_{S \subseteq N} \alpha_{S}^{c} v(S)\left(\frac{v(\{i\})}{K(v)}-\frac{1}{n}\right) .
$$

Step 2. We show that in (22), $\alpha_{S}^{c}=\alpha_{S}^{c^{\prime}}$ for each $S \subseteq N$ with $|S| \geq 2$, and thus $\alpha_{S}^{c}$, $S \subseteq N,|S| \geq 2$, does not depend on $c \in \mathbb{R}_{+}^{N}$, if $c_{i} \neq c_{j}$ for some $i, j \in N$.

With (21), we only need to consider any $c, c^{\prime} \in \mathbb{R}_{+}^{N}$ with $\sum_{k \in N} c_{k}=\sum_{k \in N} c_{k}^{\prime}$. Let $i \in N$ be a player such that $c_{i}=\min \left\{c_{k} \mid k \in N\right\}<\frac{\sum_{k \in N} c_{k}}{n}$. Suppose that there exists $j \in N$ such that $c_{i} \neq c_{j}$. Without loss of generality, we assume that $c_{j}^{\prime} \leq \frac{\sum_{k \in N} c_{k}^{\prime}}{2}$. (If $c_{j}^{\prime}>\frac{\sum_{k \in N} c_{k}^{\prime}}{2}$, we pick $h \in N \backslash\{i, j\}$ and define $c^{*} \in \mathbb{R}_{+}^{N}$ such that $c_{i}^{*}=c_{i}, c_{h}^{*}=c_{h}^{\prime}$, and $\sum_{k \in N} c_{k}^{*}=\sum_{k \in N} c_{k}$. The proof then goes in a similar way.) Then there exists a $c^{*} \in \mathbb{R}_{+}^{N}$ such that $c_{i}^{*}=c_{i}, c_{j}^{*}=c_{j}^{\prime}$, and $\sum_{k \in N} c_{k}^{*}=\sum_{k \in N} c_{k}$. Let $(N, v),(N, w) \in \mathcal{G}_{n z+}^{N}$ be two games such that $v(\{k\})=c_{k}, w(\{k\})=c_{k}^{*}$ for all $k \in N$, and $v(S)=w(S)$ for all $S \subseteq N$ with $|S| \geq 2$. Since $v(\{i\})=w(\{i\})$ and $\sum_{k \in N \backslash\{i\}} v(\{k\})=\sum_{k \in N \backslash\{i\}} w(\{k\})$, no advantageous reallocation across individuals implies

$$
\sum_{k \in N \backslash\{i\}} \psi_{k}(N, v)=\sum_{k \in N \backslash\{i\}} \psi_{k}(N, w) .
$$

With efficiency, this implies

$$
\psi_{i}(N, v)=\psi_{i}(N, w)
$$

Taking (22) into account, we obtain with $v(\{i\})=w(\{i\})$ and $K(v)=K(w)$ that

$$
\begin{aligned}
& \sum_{S \subseteq N} \alpha_{S}^{c} v(S)=\sum_{S \subseteq N} \alpha_{S}^{c^{*}} w(S) \\
\Leftrightarrow & \sum_{k \in N} \alpha_{\{k\}}^{c} v(\{k\})+\sum_{S \subseteq N:|S| \geq 2} \alpha_{S}^{c} v(S)=\sum_{k \in N} \alpha_{\{k\}}^{c^{*}} w(\{k\})+\sum_{S \subseteq N:|S| \geq 2} \alpha_{S}^{c^{*}} w(S) \\
\Leftrightarrow & \sum_{k \in N} \alpha_{\{k\}}^{c} v(\{k\})+\sum_{S \subseteq N:|S| \geq 2} \alpha_{S}^{c} v(S)=\sum_{k \in N} \alpha_{\{k\}}^{c^{*}} w(\{k\})+\sum_{S \subseteq N:|S| \geq 2} \alpha_{S}^{c^{*}} v(S) .
\end{aligned}
$$

Pick any $T \subseteq N$ with $|T| \geq 2$. Let $\left(N, v_{1}\right),\left(N, w_{1}\right) \in \mathcal{G}_{n z+}^{N}$ be such that $v_{1}(T)=$ $w_{1}(T) \neq v(T)$, and $v_{1}(S)=v(S)$ and $w_{1}(S)=w(S)$ for all $S \subseteq N, S \neq T$. Similar to (23), we can derive an equation that the only difference with (23) is the terms of $v_{1}(T)$ and $w_{1}(T)$. Substituting this equation into $(23)$ yields $\left(v(T)-v_{1}(T)\right) \alpha_{T}^{c}=\left(v(T)-w_{1}(T)\right) \alpha_{T}^{c^{*}}$, which implies

$$
\alpha_{T}^{c}=\alpha_{T}^{c^{*}}
$$


Similarly, consider $(N, w)$ and $(N, u)$, where $(N, u) \in \mathcal{G}_{n z+}^{N}$ is such that $u(\{k\})=c_{k}^{\prime}$ for all $k \in N$, and $u(S)=w(S)$ otherwise. We obtain

$$
\alpha_{T}^{c^{\prime}}=\alpha_{T}^{c^{*}}
$$

where $c^{*}$ is as defined before. Thus, $\alpha_{T}^{c}=\alpha_{T}^{c^{\prime}}$ for each $T \subseteq N$ with $|T| \geq 2$.

Step 3. We show that in (22), there exists $\alpha_{1} \in \mathbb{R}$ such that $\sum_{k \in N} \alpha_{\{k\}}^{c} v(\{k\})=$ $\alpha_{1} \sum_{k \in N} v(\{k\})$ for all $(N, v) \in \mathcal{G}_{c+}^{N}$ and all $c \in \mathbb{R}_{+}^{N}$ with $c_{i} \neq c_{j}$ for some $i, j \in N$. Indeed, for $(N, v),(N, w) \in \mathcal{G}_{n z+}^{N}$ defined in Step 2, taking $v(S)=0$ for all $S \subseteq N$ with $|S| \geq 2$, from (23) we obtain

$$
\sum_{k \in N} \alpha_{\{k\}}^{c} v(\{k\})=\sum_{k \in N} \alpha_{\{k\}}^{c^{*}} w(\{k\})
$$

Denote $\alpha_{1}=\frac{\sum_{k \in N} \alpha_{\{k\}}^{c^{*}} w(\{k\})}{\sum_{k \in N} w(\{k\})}$. Since $\sum_{k \in N} v(\{k\})=\sum_{k \in N} w(\{k\})$, then

$$
\sum_{k \in N} \alpha_{\{k\}}^{c} v(\{k\})=\sum_{k \in N} \alpha_{\{k\}}^{c^{*}} w(\{k\})=\alpha_{1} \sum_{k \in N} w(\{k\})=\alpha_{1} \sum_{k \in N} v(\{k\}) .
$$

Similarly, applying $(23)$ to $(N, w)$ and $(N, u)$ defined in Step 2, we obtain

$$
\sum_{k \in N} \alpha_{\{k\}}^{c^{\prime}} u(\{k\})=\sum_{k \in N} \alpha_{\{k\}}^{c^{*}} w(\{k\})=\alpha_{1} \sum_{k \in N} u(\{k\}) .
$$

These two equations imply that the desired assertion holds if $\frac{v(\{i\})}{c_{i}}=1$ and $\sum_{i \in N} c_{i}$ is a fixed real number. Let $\beta \in \mathbb{R}_{+}$and $(N, v) \in \mathcal{G}_{\beta c+}^{N}=\mathcal{G}_{c+}^{N}$ be such that $\frac{v(\{i\})}{\beta c_{i}}=$ 1, i.e. $\frac{v(\{i\})}{c_{i}}=\beta$. Since $(N, v) \in \mathcal{G}_{c+}^{N}$, from above it follows that $\mathcal{G}_{c+}^{N}=\mathcal{G}_{\beta c+}^{N}$, then $\sum_{k \in N} \alpha_{\{k\}}^{c} v(\{k\})=\sum_{k \in N} \alpha_{\{k\}}^{\beta c} v(\{k\})=\alpha_{1} \sum_{k \in N} v(\{k\})$, as desired.

Step 4. The 'Step 2' and 'Step 3' considered the case that $c \in \mathbb{R}_{+}^{N}$ satisfies $c_{i} \neq c_{j}$ for some $i, j \in N$. Notice that if $c_{k}=c_{h}$ for all $k, h \in N$, each $\alpha_{S}^{c}$ in (22) does not have any bite.

Step 5. From Steps 2, 3 and 4, we can conclude that, taking $\alpha_{S}^{c}=\alpha_{S}^{N}$ for each $S \subseteq N$ with $|S| \geq 2$, and $\alpha_{\{k\}}^{c}=\alpha_{1}$ for all $k \in N$, (22) can be written as

$$
\psi_{i}(N, v)=\frac{v(N)}{n}+\left[\alpha_{1} \sum_{k \in N} v(\{k\})+\sum_{S \subseteq N:|S| \geq 2} \alpha_{S}^{N} v(S)\right]\left(\frac{v(\{i\})}{K(v)}-\frac{1}{n}\right)
$$


It is straightforward to show that $\alpha_{S}^{N}=\alpha_{T}^{N}$ for all $|S|=|T| \geq 2$ in (24) by anonymity. Therefore, setting $\alpha_{|S|}^{|N|}=\alpha_{S}^{N}$, we obtain

$$
\psi_{i}(N, v)=\frac{v(N)}{n}+\sum_{S \subseteq N} \alpha_{|S|}^{|N|} v(S)\left(\frac{v(\{i\})}{K(v)}-\frac{1}{n}\right)
$$

as desired.

\section{Proof of Theorem 4}

Before giving the technical proof of Theorem 4, we give a brief sketch. We first formulate what projection consistency requires from the relationship between $\alpha(N, v)$ and $\alpha\left(N \backslash\{j\}, v^{x}\right)$ (Step 1 below). Then, the key idea of the calculation of $\alpha(N, v)$ is to derive the coefficients $\alpha_{S}^{N}$ by considering some special games. We derive that (i) the coefficients corresponding to the grand coalition are equal for grand coalitions of the same size (Step 2), (ii) the coefficients corresponding to singletons are equal (Step 3), and (iii) the coefficients of other coalitions are zero (Step 3). After that, we derive the formula of $\alpha(N, v)$ (Step 4).

Proof of Theorem 4. Let $\alpha(N, v)=\sum_{S \subseteq N} \alpha_{S}^{N} v(S)$ be a linear function with $\alpha_{S}^{N}$ being real numbers depending on $S$ and $N$. We remark that, for any $(N, v) \in \mathcal{G}_{n z}$ with $|N| \geq 3$, $x=\psi^{\alpha}(N, v)$ and $j \in N$, since $v^{x}(\{i\})=v(\{i\})$ for all $i \in N \backslash\{j\}$, it holds that $\left(N \backslash\{j\}, v^{x}\right) \in \mathcal{G}_{n z}$. Clearly, $\psi_{i}^{\alpha}(N, v)$ can be rewritten as

$$
\psi_{i}^{\alpha}(N, v)=\frac{v(N)}{n}+\alpha(N, v)\left(\frac{v(\{i\})}{\sum_{k \in N} v(\{k\})}-\frac{1}{n}\right) .
$$

Step 1. Take any $(N, v) \in \mathcal{G}_{n z}$ with $|N| \geq 3$ and $j \in N$. For $x=\psi^{\alpha}(N, v)$ and any $i \in N \backslash\{j\}$, we have

$$
\begin{aligned}
\psi_{i}^{\alpha}\left(N \backslash\{j\}, v^{x}\right)= & \frac{v^{x}(N \backslash\{j\})}{n-1}+\alpha\left(N \backslash\{j\}, v^{x}\right)\left(\frac{v^{x}(\{i\})}{\sum_{k \in N \backslash\{j\}} v^{x}(\{k\})}-\frac{1}{n-1}\right) \\
= & \frac{v(N)-x_{j}}{n-1}+\alpha\left(N \backslash\{j\}, v^{x}\right) \frac{v(\{i\})-\frac{1}{n-1} \sum_{k \in N \backslash\{j\}} v(\{k\})}{\sum_{k \in N \backslash\{j\}} v(\{k\})} \\
= & \frac{1}{n-1}\left(v(N)-\frac{v(N)}{n}-\alpha(N, v) \frac{v(\{j\})-\frac{1}{n} \sum_{k \in N} v(\{k\})}{\sum_{k \in N} v(\{k\})}\right) \\
& +\alpha\left(N \backslash\{j\}, v^{x}\right) \frac{v(\{i\})-\frac{1}{n-1} \sum_{k \in N \backslash\{j\}} v(\{k\})}{\sum_{k \in N \backslash\{j\}} v(\{k\})}
\end{aligned}
$$




$$
\begin{aligned}
= & \frac{v(N)}{n}-\alpha(N, v) \frac{v(\{j\})-\frac{1}{n} \sum_{k \in N} v(\{k\})}{(n-1) \sum_{k \in N} v(\{k\})} \\
& +\alpha\left(N \backslash\{j\}, v^{x}\right) \frac{v(\{i\})-\frac{1}{n-1} \sum_{k \in N \backslash\{j\}} v(\{k\})}{\sum_{k \in N \backslash\{j\}} v(\{k\})},
\end{aligned}
$$

where the third equality follows by substituting $x=\psi^{\alpha}(N, v)$.

From projection consistency, $\psi_{i}^{\alpha}(N, v)=\psi_{i}^{\alpha}\left(N \backslash\{j\}, v^{x}\right)$ for all $i \in N \backslash\{j\}$, and thus we have

$$
\begin{aligned}
& \frac{v(N)}{n}+\alpha(N, v) \frac{v(\{i\})-\frac{1}{n} \sum_{k \in N} v(\{k\})}{\sum_{k \in N} v(\{k\})} \\
= & \frac{v(N)}{n}-\alpha(N, v) \frac{v(\{j\})-\frac{1}{n} \sum_{k \in N} v(\{k\})}{(n-1) \sum_{k \in N} v(\{k\})} \\
& +\alpha\left(N \backslash\{j\}, v^{x}\right) \frac{v(\{i\})-\frac{1}{n-1} \sum_{k \in N \backslash\{j\}} v(\{k\})}{\sum_{k \in N \backslash\{j\}} v(\{k\})},
\end{aligned}
$$

which is equivalent to

$$
\begin{aligned}
& \frac{\alpha(N, v)}{\sum_{k \in N} v(\{k\})}\left(v(\{i\})-\frac{\sum_{k \in N} v(\{k\})}{n}+\frac{v(\{j\})-\frac{1}{n} \sum_{k \in N} v(\{k\})}{n-1}\right) \\
= & \frac{\alpha\left(N \backslash\{j\}, v^{x}\right)}{\sum_{k \in N \backslash\{j\}} v(\{k\})}\left(v(\{i\})-\frac{\sum_{k \in N \backslash\{j\}} v(\{k\})}{n-1}\right) .
\end{aligned}
$$

Since $-\frac{\sum_{k \in N} v(\{k\})}{n}+\frac{v(\{j\})-\frac{1}{n} \sum_{k \in N} v(\{k\})}{n-1}=\frac{-(n-1) \sum_{k \in N} v(\{k\})+n v(\{j\})-\sum_{k \in N} v(\{k\})}{n(n-1)}=\frac{-\sum_{k \in N} v(\{k\})+v(\{j\})}{n-1}$, it follows that

$$
\frac{\alpha(N, v)}{\sum_{k \in N} v(\{k\})}\left(v(\{i\})-\frac{\sum_{k \in N \backslash\{j\}} v(\{k\})}{n-1}\right)=\frac{\alpha\left(N \backslash\{j\}, v^{x}\right)}{\sum_{k \in N \backslash\{j\}} v(\{k\})}\left(v(\{i\})-\frac{\sum_{k \in N \backslash\{j\}} v(\{k\})}{n-1}\right) .
$$

Since there always exists a game $(N, v) \in \mathcal{G}_{n z}$ such that $v(\{i\})-\frac{\sum_{k \in N \backslash\{j\}} v(\{k\})}{n-1} \neq 0$, then

$$
\frac{\alpha(N, v)}{\sum_{k \in N} v(\{k\})}=\frac{\alpha\left(N \backslash\{j\}, v^{x}\right)}{\sum_{k \in N \backslash\{j\}} v(\{k\})}
$$

for this game.

With the notion $K=\sum_{k \in N} v(\{k\})$, we have

$$
\frac{\alpha(N, v)}{K}=\frac{\alpha\left(N \backslash\{j\}, v^{x}\right)}{K-v(\{j\})} .
$$

By definition of $\alpha, v^{x}$ and $x=\psi^{\alpha}(N, v)$, we have

$$
\alpha\left(N \backslash\{j\}, v^{x}\right)
$$




$$
\begin{aligned}
& =\sum_{S \subset N \backslash\{j\}} \alpha_{S}^{N \backslash\{j\}} v(S)+\alpha_{N \backslash\{j\}}^{N \backslash\{j\}}\left(v(N)-x_{j}\right) \\
& =\sum_{S \subset N \backslash\{j\}} \alpha_{S}^{N \backslash\{j\}} v(S)+\alpha_{N \backslash\{j\}}^{N \backslash\{j\}}\left(\frac{n-1}{n} v(N)-\sum_{S \subseteq N} \alpha_{S}^{N} v(S) \frac{v(\{j\})-\frac{K}{n}}{K}\right) .
\end{aligned}
$$

Step 2. We show that $\alpha_{N \backslash\{i\}}^{N \backslash\{i\}}=\alpha_{N \backslash\{j\}}^{N \backslash\{j\}}$ for all $i, j \in N$. To show this, consider a game $(N, v) \in \mathcal{G}_{n z}$ with $i, j \in N$ such that $v(\{i\})=v(\{j\})$ and $v(\{i\})-\frac{\sum_{k \in N \backslash\{j\}} v(\{k\})}{n-1} \neq 0$ (It is possible since $|N| \geq 3)$. From (26) we have $\alpha\left(N \backslash\{i\}, v^{x}\right)=\alpha\left(N \backslash\{j\}, v^{x}\right)$. That is, with $(27)$

$$
\begin{aligned}
& \sum_{S \subset N \backslash\{i\}} \alpha_{S}^{N \backslash\{i\}} v(S)+\alpha_{N \backslash\{i\}}^{N \backslash\{i\}}\left(\frac{n-1}{n} v(N)-\sum_{S \subseteq N} \alpha_{S}^{N} v(S) \frac{v(\{i\})-\frac{K}{n}}{K}\right) \\
= & \sum_{S \subset N \backslash\{j\}} \alpha_{S}^{N \backslash\{j\}} v(S)+\alpha_{N \backslash\{j\}}^{N \backslash\{j\}}\left(\frac{n-1}{n} v(N)-\sum_{S \subseteq N} \alpha_{S}^{N} v(S) \frac{v(\{j\})-\frac{K}{n}}{K}\right) .
\end{aligned}
$$

The coefficients of the term $v(N)$ must be the same on both sides of (28), that is

$$
\alpha_{N \backslash\{i\}}^{N \backslash\{i\}}\left(\frac{n-1}{n}-\alpha_{N}^{N} \frac{v(\{i\})-\frac{K}{n}}{K}\right)=\alpha_{N \backslash\{j\}}^{N \backslash\{j\}}\left(\frac{n-1}{n}-\alpha_{N}^{N} \frac{v(\{j\})-\frac{K}{n}}{K}\right) .
$$

No matter what number $\alpha_{N}^{N}$ is, there is a game such that $\frac{n-1}{n}-\alpha_{N}^{N} \frac{v(\{i\})-\frac{K}{n}}{K} \neq 0 .{ }^{7}$ Thus,

$$
\alpha_{N \backslash\{i\}}^{N \backslash\{i\}}=\alpha_{N \backslash\{j\}}^{N \backslash\{j\}}, \quad \text { for all } i, j \in N .
$$

Step 3. We derive $\alpha_{T}^{N \backslash\{k\}}$ for any $k \in N$ and $T \subset N \backslash\{k\}$, as follows. Using (29), it follows from $(28)$ and the fact that $v(\{i\})=v(\{j\})$, that

$$
\sum_{S \subset N \backslash\{i\}} \alpha_{S}^{N \backslash\{i\}} v(S)=\sum_{S \subset N \backslash\{j\}} \alpha_{S}^{N \backslash\{j\}} v(S) .
$$

To derive $\alpha_{T}^{N \backslash\{k\}}$, we consider two cases with respect to $|T|, T \subset N$ :

(i) For $T \subset N$ with $|T| \geq 2$, there exist $i, j \in N$ with $T \subset N \backslash\{i\}$ and $j \in T$. Clearly, the term $v(T)$ only appears in the left-hand side of (30), and thus it must be that $\alpha_{T}^{N \backslash\{i\}}=0$. (We see this by taking two games that only differ in the worth of $T$.) Similarly, $\alpha_{T}^{N \backslash\{j\}}=0$ for all $T \subset N \backslash\{j\}$ with $i \in T$ and $|T| \geq 2$.

\footnotetext{
${ }^{7}$ For example, a game $(N, v)$ such that there is an $i_{0} \in N$ with $v\left(\left\{i_{0}\right\}\right)=2$ and $v(\{j\})=1$ for all $j \in N \backslash\left\{i_{0}\right\}$ will do the job if $\alpha_{N}^{N} \neq(n+1)$, and a game $(N, v)$ such that there are $i_{0}, i_{1} \in N$ with $v\left(\left\{i_{0}\right\}\right)=v\left(\left\{i_{1}\right\}\right)=2$ and $v(\{j\})=1$ for all $j \in N \backslash\left\{i_{0}, i_{1}\right\}$ will do the job otherwise.
} 
Since $i, j \in N$ can be arbitrary two players, we obtain

$$
\alpha_{T}^{N \backslash\{k\}}=0,
$$

for all $T \subset N$ with $|T| \geq 2$.

(ii) Consider $T \subset N$ with $|T|=1$. Since (30) holds under the condition $v(\{i\})=v(\{j\})$, it must be $\alpha_{\{j\}}^{N \backslash\{i\}} v(\{j\})=\alpha_{\{i\}}^{N \backslash\{j\}} v(\{i\})$. (We see this by taking two games that only differ in the worths of $\{i\}$ and $\{j\}$ and using (31).) Since $v(\{i\})=v(\{j\}) \neq 0$, then $\alpha_{\{j\}}^{N \backslash\{i\}}=\alpha_{\{i\}}^{N \backslash\{j\}}$. Similarly, it is clear that $\alpha_{\{k\}}^{N \backslash\{i\}}=\alpha_{\{k\}}^{N \backslash\{j\}}$ for all $k \in N \backslash\{i, j\}$.

Since $i, j \in N$ can be arbitrary two players, then $\alpha_{\{j\}}^{N \backslash\{i\}}=\alpha_{\{j\}}^{N \backslash\{k\}}=\alpha_{\{k\}}^{N \backslash j j\}}=\alpha_{\{k\}}^{N \backslash\{i\}}$ for all $k \in N \backslash\{i, j\}$. Therefore,

$$
\alpha_{\{i\}}^{N \backslash\{k\}}=\alpha_{\{j\}}^{N \backslash\{k\}},
$$

for all $i, j \in N \backslash\{k\}$ and all $k \in N$.

Step 4. Now, we can derive the desired assertion. Using (32), we denote $\beta^{n-1}=\alpha_{i}^{N \backslash\{k\}}$ for all $i \in N \backslash\{k\}$. Plugging (31) and (32) into the second line of (27), we have that for all $j \in N$,

$$
\begin{aligned}
\alpha\left(N \backslash\{j\}, v^{x}\right) & =\beta^{n-1} \sum_{k \in N \backslash\{j\}} v^{x}(\{k\})+\alpha_{N \backslash\{j\}}^{N \backslash\{j\}} v^{x}(N \backslash\{j\}) \\
& =\beta^{n-1}(K(v)-v(\{j\}))+\alpha_{N \backslash\{j\}}^{N \backslash j j\}} v^{x}(N \backslash\{j\}) .
\end{aligned}
$$

Consider a game $\left(N^{\prime}, v^{\prime}\right) \in \mathcal{G}_{n z}$ with $N^{\prime}=N \cup\{j\}, j \notin N,\left(v^{\prime}\right)^{x}=v$. Similar as above, denoting $\beta^{n}=\alpha_{i}^{N}$ for all $i \in N$, we obtain

$$
\alpha(N, v)=\beta^{n} K(v)+\alpha_{N}^{N} v(N) .
$$

Therefore, (26) can be written as

$$
\begin{aligned}
\beta^{n}+\frac{\alpha_{N}^{N} v(N)}{K} & =\beta^{n-1}+\frac{\alpha_{N \backslash j j\}}^{N \backslash\{j\}} v^{x}(N \backslash\{j\})}{K-v(\{j\})} \\
& =\beta^{n-1}+\frac{\alpha_{N \backslash\{j\}}^{N \backslash\{j\}}}{K-v(\{j\})}\left(\frac{n-1}{n} v(N)-\alpha_{N}^{N} v(N) \frac{v(\{j\})-\frac{K}{n}}{K}-\beta^{n}\left(v(\{j\})-\frac{K}{n}\right)\right),
\end{aligned}
$$

where the second equality follows from (25). 
Considering any game $(N, v) \in \mathcal{G}_{n z}$ such that $K(v)=n$ and $v(\{j\})=1$ (for example by taking a game $(N, v),|N| \geq 3$, such that $v\left(\left\{i_{0}\right\}\right)=0.5, v\left(\left\{i_{1}\right\}\right)=1.5$, and $v(\{k\})=1$ for all $\left.k \in N \backslash\left\{i_{0}, i_{1}\right\}\right)$, we obtain $\beta^{n}+\frac{\alpha_{N}^{N} v(N)}{n}=\beta^{n-1}+\frac{\alpha_{N \backslash\{j\}}^{N \backslash\{j\}} v(N)}{n}$. Since $v(N)$ can take any number, it must be that $\beta^{n-1}=\beta^{n}$ (by taking a game with $v(N)=0$ ) and then $\alpha_{N}^{N}=\alpha_{N \backslash\{j\}}^{N \backslash\{j\}}$ (by taking a game with $v(N)>0$ ). Hence, the above equation, for any $(N, v) \in \mathcal{G}_{n z}$, can be written as follows:

$$
\frac{\alpha_{N}^{N} v(N)}{K}=\frac{\alpha_{N}^{N}}{K-v(\{j\})}\left(\frac{n-1}{n} v(N)-\alpha_{N}^{N} v(N) \frac{v(\{j\})-\frac{K}{n}}{K}-\beta^{n}\left(v(\{j\})-\frac{K}{n}\right)\right),
$$

which implies that

$$
(K-v(\{j\})) \alpha_{N}^{N} v(N)=K \alpha_{N}^{N}\left(\frac{n-1}{n} v(N)-\alpha_{N}^{N} v(N) \frac{v(\{j\})-\frac{K}{n}}{K}-\beta^{n}\left(v(\{j\})-\frac{K}{n}\right)\right) .
$$

There are two parameters $\alpha_{N}^{N}$ and $\beta^{n}$ to be determined in (34). We distinguish the following two cases:

(i) If $\alpha_{N}^{N}=0$, then $\beta^{n}$ can take any real number, and thus (6) gives an affine combination of the ED value and ESD value.

(ii) If $\alpha_{N}^{N} \neq 0$, then

$$
(K-v(\{j\})) v(N)=K \frac{n-1}{n} v(N)-\left(\alpha_{N}^{N} v(N)+K \beta^{n}\right)\left(v(\{j\})-\frac{K}{n}\right) .
$$

It follows from (35) that $v(N)\left(v(\{j\})-\frac{K}{n}\right)=\left(\alpha_{N}^{N} v(N)+K \beta^{n}\right)\left(v(\{j\})-\frac{K}{n}\right)$. Considering any game with $v(\{j\})-\frac{K}{n} \neq 0$, we obtain

$$
\alpha_{N}^{N} v(N)+K \beta^{n}=v(N)
$$

Since this must hold for any $v(N)$, it follows that $\beta^{n}=0$ (by taking $v(N)=0$ ), and thus $\alpha_{N}^{N}=1$ (by taking $v(N)>0$ ). Hence, (6) gives the PD value.

We conclude from (33), and Cases (i) and (ii), that if $\psi^{\alpha}$ satisfies projection consistency, then $\alpha(N, v)=\beta \sum_{k \in N} v(\{k\}), \beta \in \mathbb{R}$, or $\alpha(N, v)=v(N)$. Therefore,

$$
\psi^{\alpha}(N, v)= \begin{cases}\beta E S D(N, v)+(1-\beta) E D(N, v), & \text { if } \alpha(N, v)=\beta \sum_{k \in N} v(\{k\}), \\ P D(N, v), & \text { if } \alpha(N, v)=v(N) .\end{cases}
$$


The above assertion holds for $|N| \geq 3$. We now turn to the case $|N|=2$. We already know that $\beta^{\left|N^{\prime}\right|-1}=\beta^{\left|N^{\prime}\right|}$ and $\alpha_{N^{\prime}}^{N^{\prime}}=\alpha_{N^{\prime}}^{N^{\prime} \backslash\{j\}}$ for all $\left|N^{\prime}\right| \geq 3$ and all $j \in N^{\prime}$. Meanwhile, Cases (i) and (ii) hold for $\left|N^{\prime}\right| \geq 3$. Specifically, taking $\left|N^{\prime}\right|=3$, we obtain that $\alpha(N, v)=v(N)$ or $\alpha(N, v)=\beta \sum_{k \in N} v(\{k\})$ for $|N|=\left|N^{\prime}\right|-1=2$, which immediately yields the desired assertion.

Proof of Theorem 3. As mentioned in the main text, since the class of $\alpha$-mollified values is contained in the class of $\alpha$-mollified generalized values, and the resulting values in Theorem 4 are also members in the family of $\alpha$-mollified values, we obtain Theorem 3 as a corollary of Theorem 4. A direct proof of Theorem 3 can be given by omitting Step 2 and Step 3 of the proof of Theorem 4 .

Proof of Theorem 5. In view of (7), we have

$$
\begin{aligned}
& \sum_{\pi \in \Pi(N)} \frac{1}{n !} \eta_{i}^{\pi, \alpha} \\
= & \sum_{\substack{\pi \in \Pi(N): \\
\pi(i)=1}} \frac{1}{n !}\left[v(\{i\})+\sum_{j \in N: \pi(j)>\pi(i)} \frac{v\left(S_{\pi}^{j}\right)-v\left(S_{\pi}^{j} \backslash\{j\}\right)-\frac{v(\{j\}) \alpha(N, v)}{\sum_{k \in N} v(\{k\})}}{\pi(j)-1}\right]+\sum_{\substack{\pi \in \Pi(N): \\
\pi(i)=n}} \frac{1}{n !} \frac{v(\{i\}) \alpha(N, v)}{\sum_{k \in N} v(\{k\})} \\
& +\sum_{\substack{\pi \in \Pi(N): \\
\pi(i) \neq 1, n}} \frac{1}{n !}\left[\frac{v(\{i\}) \alpha(N, v)}{\sum_{k \in N} v(\{k\})}+\sum_{j \in N: \pi(j)>\pi(i)} \frac{v\left(S_{\pi}^{j}\right)-v\left(S_{\pi}^{j} \backslash\{j\}\right)-\frac{v(\{j\}) \alpha(N, v)}{\sum_{k \in N} v(\{k\})}}{\pi(j)-1}\right] \\
= & \frac{v(\{i\})}{n}+\frac{n-1}{n} \frac{v(\{i\}) \alpha(N, v)}{\sum_{k \in N} v(\{k\})}+\frac{1}{n !} \sum_{\pi \in \Pi(N)} \sum_{j \in N: \pi(j)>\pi(i)} \frac{v\left(S_{\pi}^{j}\right)-v\left(S_{\pi}^{j} \backslash\{j\}\right)-\frac{v(\{j\}) \alpha(N, v)}{\sum_{k \in N} v(\{k\})}}{\pi(j)-1},
\end{aligned}
$$

where the second equality holds since there are $(n-1)$ ! (respectively $(n-1)(n-1) !)$ permutations such that $i$ has (respectively does not have) the first position, the same for the last position.

Note that for each coalition $S \subseteq N$ with $j \in S$, there are $(n-s) !(s-1)$ ! permutations such that the first $s$ players are exactly the members of $S$ and $j$ has the $s$ th position. Hence, the third term of the right-hand side in (37) can be rewritten as follows:

$$
\sum_{j \in N \backslash\{i\}} \sum_{S \subseteq N: i, j \in S} \frac{v(S)-v(S \backslash\{j\})-\frac{v(\{j\}) \alpha(N, v)}{\sum_{k \in N} v(\{k\})}}{s-1} \frac{(n-s) !(s-1) !}{n !}
$$




$$
=\sum_{j \in N \backslash\{i\}} \sum_{S \subseteq N: i, j \in S} \frac{(n-s) !(s-2) !}{n !}[v(S)-v(S \backslash\{j\})]-\sum_{j \in N \backslash\{i\}}\left[\frac{v(\{j\}) \alpha(N, v)}{\sum_{k \in N} v(\{k\})} \sum_{S \subseteq N: i, j \in S} \frac{(n-s) !(s-2) !}{n !}\right]
$$

We can rewrite the first term as

$$
\begin{aligned}
& \sum_{j \in N \backslash\{i\}} \sum_{S \subseteq N: i, j \in S} \frac{(n-s) !(s-2) !}{n !}[v(S)-v(S \backslash\{j\})] \\
= & \sum_{S \subseteq N:|S| \geq 2, i \in S} \sum_{j \in S \backslash\{i\}} \frac{(n-s) !(s-2) !}{n !}[v(S)-v(S \backslash\{j\})] \\
= & \sum_{s=2}^{n} \sum_{S \subseteq N: i \in S,|S|=s} \frac{(s-1) !(n-s) !}{n !} v(S)-\sum_{s=1}^{n-1} \sum_{S \subseteq N: i \in S,|S|=s} \frac{(s-1) !(n-s) !}{n !} v(S) \\
= & \frac{(n-1) !(n-n) !}{n !} v(N)-\frac{(1-1) !(n-1) !}{n !} v(\{i\}) \\
= & \frac{v(N)}{n}-\frac{v(\{i\})}{n} .
\end{aligned}
$$

Since

$$
\sum_{S \subseteq N: i, j \in S} \frac{(n-s) !(s-2) !}{n !}=\sum_{s=2}^{n} \frac{(n-s) !(s-2) !}{n !} \frac{(n-2) !}{(n-s) !(s-2) !}=(n-1) \frac{(n-2) !}{n !}=\frac{1}{n},
$$

we obtain from (37) and (38) that

$$
\begin{aligned}
\sum_{\pi \in \Pi(N)} \frac{1}{n !} \eta_{i}^{\pi, \alpha} & =\frac{v(\{i\})}{n}+\frac{n-1}{n} \frac{v(\{i\}) \alpha(N, v)}{\sum_{k \in N} v(\{k\})}+\frac{v(N)}{n}-\frac{v(\{i\})}{n}-\frac{1}{n} \frac{\sum_{j \in N \backslash\{i\}} v(\{j\}) \alpha(N, v)}{\sum_{k \in N} v(\{k\})} \\
& =\frac{v(\{i\})}{\sum_{k \in N} v(\{k\})} \alpha(N, v)+\frac{1}{n}(v(N)-\alpha(N, v))
\end{aligned}
$$

as desired.

\section{Acknowledgement}

The authors would like to thank Daniel Reem for helpful comments on a previous version of the paper. Zhengxing Zou thanks the financial support of the National Natural Science Foundation of China (Grant Nos. 71771025, 71801016). Yukihiko Funaki is supported by JSPS KAKENHI Grant Numbers JP17H02503 and JP18KK0046. 


\section{References}

Aczél, J., Erdös, P., 1965. The non-existence of a Hamel-basis and the general solution of Cauchy's functional equation for nonnegative numbers. Publicationes Mathematicae Debrecen 12, 259-263.

Béal, S., Ferrières, S., Rémila, E., Solal, P., 2018. The proportional Shapley value and applications. Games and Economic Behavior 108, 93-112.

Besner, M., 2019. Axiomatizations of the proportional Shapley value. Theory and Decision 86(2), 161-183.

Calleja, P., Llerena, F., 2017. Rationality, aggregate monotonicity and consistency in cooperative games: some (im)possibility results. Social Choice and Welfare 48, 197-220.

Calleja, P., Llerena, F., 2019. Path monotonicity, consistency and axiomatizations of some weighted solutions. International Journal of Game Theory 48(1), 287-310.

Casajus, A., 2014. Potential, value, and random partitions. Economics Letters 125(2), $164-166$.

Driessen, T., 1991. A survey of consistency properties in cooperative game theory. SIAM review 33(1), 43-59.

Driessen, T., Funaki, Y., 1991. Coincidence of and collinearity between game theoretic solutions. OR-Spektrum 13(1), 15-30.

Dutta, B., Ray, D., 1989. A concept of egalitarianism under participation constraints. Econometrica 57(3), 615-635.

Ertemel, S., Kumar, R., 2018. Proportional rules for state contingent claims. International Journal of Game Theory 47(1), 229-246.

Funaki, Y., Yamato, T., 2001. The core and consistency properties: a general characterisation. International Game Theory Review 3(2-3), 175-187.

Giménez-Gómez, J., Peris, J., 2014. A proportional approach to claims problems with a guaranteed minimum. European Journal of Operational Research 232(1), 109-116. 
Hamel, G., 1905. Eine Basis aller Zahlen und die unstetigen Lösungen der Funktionalgleichung: $f(x+y)=f(x)+f(y)$. Mathematische Annalen 60(3), 459-462.

Hart, S., Mas-Colell, A., 1989. Potential, value, and consistency. Econometrica 57(3), 589614.

Hou, D., Lardon, A., Sun, P., Sun, H., 2019. Procedural and optimization implementation of the weighted ENSC value. Theory and Decision 87(2), 171-182.

Hwang, Y., Li, J., Hsiao, Y., 2005. A dynamic approach to the Shapley value based on associated games. International Journal of Game Theory, 33(4), 551-562.

Kuczma, M., 2009. An Introduction to the Theory of Functional Equations and Inequalities: Cauchy's Equation and Jensen's Inequality, 2nd. Birkhöuser, Basel.

Malawski, M. (2013). "Procedural" values for cooperative games. International Journal of Game Theory, 42(1), 305-324.

Moulin, H., 1987. Equal or proportional division of a surplus, and other methods. International Journal of Game Theory 16(3), 161-186.

O'Neill, B., 1982. A problem of rights arbitration from the Talmud. Mathematical Social Sciences 2(4), 345-371.

Sahoo, P., Kannappan, P., 2011. Introduction to Functional Equations. CRC Press, Boca Raton.

Thomson, W., 2003. Axiomatic and game-theoretic analysis of bankruptcy and taxation problems: a survey. Mathematical Social Sciences 45(3), 249-297.

Thomson, W., 2008. The two-agent claims-truncated proportional rule has no consistent extension: A constructive proof. Economics Letters 98(1), 59-65.

Thomson, W., 2011. Consistency and its converse: an introduction. Review of Economic Design 15(4), 257-291.

Thomson, W., 2013. A characterization of a family of rules for the adjudication of conflicting claims. Games and Economic Behavior 82, 157-168. 
Thomson, W., 2015a. Axiomatic and game-theoretic analysis of bankruptcy and taxation problems: an update. Mathematical Social Sciences 74, 41-59.

Thomson, W., 2015b. For claims problems, compromising between the proportional and constrained equal awards rules. Economic Theory 60(3), 495-520.

Thomson, W., 2019. How to Divide when there Isn't Enough: from Aristotle, the Talmud, and Maimonides to the Axiomatics of Resource Allocation, Cambridge University Press, pp. 270-307.

van den Brink, R., 2007. Null or nullifying players: The difference between the Shapley value and equal division solutions. Journal of Economic Theory 136(1), 767-775.

van den Brink, R., Chun, Y., Funaki, Y., Park, B., 2016. Consistency, population solidarity, and egalitarian solutions for TU-games. Theory and Decision 81(3), 427-447.

van den Brink, R., Funaki, Y., 2009. Axiomatizations of a class of equal surplus sharing solutions for TU-games. Theory and Decision 67(3), 303-340.

Vorob'ev, N., Liapunov, A., 1998. The proper Shapley value. Game Theory and Applications 4, 155-159.

Wang, W., Sun, H., van den Brink, R. Xu, G., 2019. The family of ideal values for cooperative games. Journal of Optimization Theory and Applications 180(3), 1065-1086.

Zou, Z., van den Brink, R., Chun, Y., Funaki, Y., 2019. Axiomatizations of the proportional division value. Tinbergen Institute Discussion Paper 19-072/II.

Zou, Z., van den Brink, R., Funaki, Y., 2020. Sharing the surplus and proportional values. Tinbergen Institute Discussion Paper 20-014/II. 